\title{
The Detection and Characterization of a Nontransiting Planet by Transit Timing Variations
}

\author{
David Nesvorný ${ }^{1 *}$, David M. Kipping ${ }^{2,3}$, Lars A. Buchhave ${ }^{4,5}$, \\ Gáspár Á. Bakos ${ }^{6}$, Joel Hartman ${ }^{6}$, Allan R. Schmitt ${ }^{7}$ \\ ${ }^{1}$ Department of Space Studies, Southwest Research Institute, Boulder, CO 80302, USA \\ ${ }^{2}$ Harvard-Smithsonian Center for Astrophysics, Cambridge, MA 02138, USA \\ ${ }^{3}$ NASA Carl Sagan Fellow \\ ${ }^{4}$ Niels Bohr Institute, University of Copenhagen, DK-2100, Copenhagen, Denmark \\ ${ }^{5}$ Centre for Star and Planet Formation, Natural History Museum of Denmark \\ University of Copenhagen, DK-1350, Copenhagen, Denmark \\ ${ }^{6}$ Department of Astrophysical Sciences, Princeton University, Princeton, NJ 05844, USA \\ ${ }^{7}$ Citizen Science \\ ** To whom correspondence should be addressed; E-mail: davidn@boulder.swri.edu.
}

The Kepler Mission is monitoring the brightness of $\sim 150,000$ stars searching for evidence of planetary transits. As part of the "Hunt for Exomoons with Kepler" (HEK) project, we report a planetary system with two confirmed planets and one candidate planet discovered using the publicly available data for KOI-872. Planet $\mathbf{b}$ transits the host star with a period $P_{b}=33.6 \mathbf{d}$ and exhibits large transit timing variations indicative of a perturber. Dynamical modeling uniquely detects an outer nontransiting planet c near the 5:3 resonance $\left(P_{c}=57.0 \mathrm{~d}\right)$ of mass $\mathbf{0 . 3 7}$ times that of Jupiter. Transits of a third planetary candidate are also found: a 1.7-Earth radius super-Earth with a $6.8 \mathrm{~d}$ period. Our analysis indicates a system with nearly coplanar and circular orbits, reminiscent of the orderly arrangement within the solar system. 
If a planet's orbit is viewed nearly edge-on, the planet may transit over the disk of its host star and periodically block a small fraction of the starlight. The planet's presence is then revealed by a small and repetitive decrease of the host star's brightness during transits. The transit light curve is characterized by the time of transit minimum $\tau$, the transit depth $\delta$, the total duration $T_{14}$, and the partial duration $T_{23}(1)$. A precise measurement of these terms allows an observer to infer the physical properties of the system, such as the radius ratio, $p=R_{P} / R_{*}$, transit impact parameter, $b_{P}$, and scaled semi-major axis, $a_{P} / R_{*}$, where $R_{*}$ is the star's physical radius and the subscript $P$ denotes "planet".

For a planet following a strictly Keplerian orbit, the spacing, timing and other properties of the transit light curve should be unchanging in time. Several effects, however, can produce deviations from the Keplerian case so that the spacing of $\tau$ is not strictly periodic and/or $T_{14}$ varies from transit to transit. Such changes are known as transit timing variations (TTVs) and transit duration variations (TDVs), respectively. TTVs are particularly sensitive to gravitational perturbations from additional planets orbiting the host star (2-4) and distant large moons orbiting the transiting planet (5-7).

As part of the "Hunt for Exomoons with Kepler" (HEK) project (8), we analyzed the publicly available Kepler data up to Quarter 6 (Q6; released on January 7, 2012). At the time of writing, the 33.6-day-period planetary candidate Kepler-object-of-interest 872.01 (KOI-872.01) is the only known candidate in the system (9). The candidate was identified through HEK's target selection procedure as a high priority object because of the presence of visual transit anomalies and the dynamical capacity to host a moon.

We detrended the raw Kepler photometry of KOI-872, covering 15 transits, using a harmonic filter and an exponential decay ramp correction (10). We tested several models to explain the photometry using the multimodal nested sampling algorithm $\operatorname{MultiNest}(11,12)$, designed to compute the Bayesian evidence for each model. The favored model was found to be that of a planet undergoing TTVs (each transit has a unique $\tau$ but common parameters for all other 
terms; model $\mathcal{M}_{T}$ ). This model is preferred over that of a planet on a linear ephemeris, $\mathcal{M}_{P}$, at a confidence of $43.7 \sigma$ (Fig. 1).

We computed TTVs relative to the linear ephemeris derived from $\mathcal{M}_{P}\left(P_{b}=33.60134 \pm\right.$ $0.00021 \mathrm{~d}$ and $\tau_{0}=2455053.2826 \pm 0.0014 \mathrm{BJD}_{\mathrm{UTC}}$, where $\mathrm{BJD}_{\mathrm{UTC}}$ is understood to be barycentric Julian date in coordinated universal time). The results (Fig. 2) indicate that KOI872.01 exhibits large ( 2 hours) and complex TTVs with a dominant period of about $\simeq 5.6$ transit cycles $(\simeq 190 \mathrm{~d})$. These are among the largest TTVs ever detected. A model including TDVs is disfavored relative to the TTV-only model at a confidence of $17.5 \sigma$.

Because a moon should induce TDVs and TTVs, the lack of TDVs does not favor a moon hypothesis. Further, Q4-Q6 photometry show complex stellar activity, which may be responsible for the initially identified visual anomalies. In support of these arguments, we found a planet-with-moon model $\left(\mathcal{M}_{M}\right)$ inadequate to explain the measured TTVs (Fig. $\left.2 \mathrm{c}\right)$.

A distant stellar companion or secular/resonant perturbations from a planetary companion also cannot explain KOI-872.01's TTVs, because these effects would produce sinusoidal patterns with a very long period (13). Moreover, other known TTV effects, such as parallax effects (14), the Applegate effect (15), and stellar proper motion (16), can be ruled out because they are unable to produce such a large TTV amplitude.

We applied the TTV inversion method described in (17) to test whether the observed TTVs are consistent with short-period perturbations from a planetary companion and whether a unique set of parameters can be derived to describe the physical and orbital properties of that companion. The short-period perturbations are small variations around the mean Keplerian orbit of a planet with characteristic periods comparable to the planet's orbital period. The inversion method is based on perturbation theory, which greatly speeds up the computation of the timing and duration of individual transits (18).

We tested orbits with periods between 1 day and 10 years, including the cases of highly eccentric and/or retrograde planets (19). The identified solutions were fine tuned using a precise 
$N$-body integrator. We used the downhill simplex method to search for the minimum of $\chi^{2}=$ $\sum_{j=-1}^{n}\left(\delta t_{\mathrm{O}, j}-\delta t_{\mathrm{C}, j}\right)^{2} / \sigma_{j}^{2}$, where $n=15$ is the number of transits, $\delta t_{\mathrm{O}, j}$ and $\delta t_{\mathrm{C}, j}$ are the observed and calculated TTVs, and $\sigma_{j}$ is the uncertainty of $\delta t_{\mathrm{O}, j}$. Local minima in $\chi^{2}$ were tested for physical plausibility, including a stability test where the orbits were tracked over $10^{9} \mathrm{yr}$ with a symplectic integrator (20).

All except two solutions can be ruled out because they are either dynamically unstable or have $\chi_{\min }^{2}>60$. The best-fit solution (hereafter s1) fits the data extremely well: $\chi_{\min , \mathrm{s} 1}^{2}=3.4$ for $n-m=15-7=8$ degrees of freedom (DOF), where $m=7$ parameters of the perturbing planet are the mass ratio $M_{c} / M_{*}$, period $P_{c}$, eccentricity $e_{c}$, inclination $I_{c}$, nodal longitude $\Omega_{c}$, pericenter longitude $\varpi_{c}$, and mean longitude $\lambda_{c}$. The inclination $I$ is defined relative to the reference plane that is $90^{\circ}$ tilted to the sky plane, and rotated so that $\Omega_{b}=270^{\circ}$ (all longitudes measured relative to the line of sight; hereafter, transit reference system). The orbital inclination of KOI-872.01 relative to the transit plane is $I_{b}=0.96^{\circ}$, as determined from the transit fit.

The second best-fit solution (s2) has $\chi_{\min , \mathrm{s} 2}^{2}=20.3$. It corresponds to a planet with $M_{c} \simeq$ $1.8 \times 10^{-3} M_{*}, P_{c} \simeq 81.7$ days (i.e. $P_{c} / P_{b}=2.43$, just inside the 5:2 resonance), $e_{c} \simeq 0.03$ and $I_{c} \simeq 10^{\circ}$. s2 can be ruled out because the goodness-of-fit for the TTVs is significantly worse at $\Delta \chi^{2}=16.9$; also, as $I_{c}$ is relatively large, s2 implies strong TDVs that are inconsistent with the observations (Fig. 2 $2 \mathrm{c})(21)$.

Therefore the transit variations of KOI-872.01 can only be fit by s1. This was by no means expected or guaranteed because the short-periodic TTVs produced by the interacting planets represent only a very specific subset of astrophysical signals. This can be demonstrated by scrambling the TTV datapoints and applying the TTV inversion method to the scrambled data. We were unable to find a plausible planet solution for any of the attempted (thousand) trials with the scrambled data. This is a strong indication that KOI-872 is a real system of at least two planets (22) (Table 1).

The scaled mass of KOI-872.02 inferred from TTVs of KOI-872.01 is $M_{c} / M_{*}=3.97 \times$ 
$10^{-4}$. With $M_{*} \simeq 0.9 M_{\odot}$, obtained from spectroscopy, this gives $M_{c} \simeq 0.37 M_{J}$, or $\sim 1.3$ Saturn masses. The mass of KOI-872.01, $M_{b}$, cannot be constrained from TTVs because the short-periodic TTVs are practically independent of $M_{b}(3,18)$. The stability requirements imply that $M_{b}<6 M_{J}$, because the orbits are dynamically unstable with a more massive transiting body. This mass limit along with our vetting analysis (10) confirm KOI-872.01 and the perturber as being genuine planets, henceforth referred to KOI-872b (corresponding to KOI-872.01) and KOI-872c.

The period ratio, $P_{c} / P_{b}=1.697$ indicates that the two planets are just outside the $5: 3$ resonance. This may be a relatively common configuration probably related to the radial migration of planets in the protoplanetary nebula (23). The resonant angle, $5 \lambda_{c}-3 \lambda_{b}-2 \varpi_{c}$, circulates in the retrograde sense with the period of $\simeq 2$ yr. The dominant TTV period $(\simeq 5.6$ transit cycles; Fig. 2) comes from the relatively distant 2:1 and 3:2 terms (periods $\simeq 190 \mathrm{yr}$ ).

The orbital eccentricities are $e_{b}<0.02$ and $e_{c} \simeq 0.015$. The nearly circular orbits probably indicate that the two planets formed at, or migrated to, their present orbits without suffering any strong dynamical instability. The two planets also exhibit nearly but not exactly coplanar orbits. The orbital inclination of KOI-872c with respect to the transit plane is $I_{c}<4.5^{\circ}(99 \%$ confidence interval) with the best TTV fit indicating $I_{c}=2.6^{\circ}$. The best-fit inclination value and $\Omega_{c} \simeq 298^{\circ}$ obtained from the TTVs suggest the planet just avoids transiting. A search for the transits of KOI-872c indeed yielded no events, implying that $I_{c}>1^{\circ}$.

Although no transits of KOI-872c were detected, we do detect a 9- $\sigma$ transit signal on a short period of $6.8 \mathrm{~d}$. The transit corresponds to a $1.7 R_{\oplus}$ Super-Earth, which we refer to as KOI872.03. Because of the probable low-mass nature of this body, the expected TTVs induced on KOI-872b would be around $1 \mathrm{~s}$ in amplitude, too small to detect with our data. Further, the TTVs of KOI-872.03 itself are estimated to be $\sim 10 \mathrm{~s}$, also undetectable. Without TTVs, we are unable to confirm that KOI-872.03 orbits the same star as KOI-872b and KOI-872c, as opposed to a blended background star. However, our analysis finds that KOI-872.03's light curve derived 
stellar density is consistent with that of KOI-872b for both planets on near-circular orbits.

We predict that the radial velocity (RV) measurements of KOI-872 should reveal at least two basic periods. The RV term with a $57.0 \mathrm{~d}$ period, corresponding to KOI-872c, should have $K_{c} \simeq 20 \mathrm{~m} \mathrm{~s}^{-1}$ half-amplitude. The amplitude of the $33.6 \mathrm{~d}$ period term, corresponding to KOI-872b, is uncertain because we do not have a good constraint on $M_{b}$. The $6.8 \mathrm{~d}$ term, corresponding to KOI-872.03, will be difficult to detect because $K_{3} \sim 1-2 \mathrm{~m} \mathrm{~s}^{-1}$ (assuming Earth-like density).

To gain insights into the system's dynamical behavior we numerically integrated the planetary orbits starting from the best-fit solution (Fig. 3). The semi-major axis of KOI-872b experiences short-period variations, related to the TTVs, with an amplitude of $\simeq 5 \times 10^{-4} \mathrm{AU}$. The eccentricities undergo anti-correlated oscillations, as dictated by the angular momentum conservation, around means $\bar{e}_{b}=0.013$ and $\bar{e}_{c}=0.012$, with a period of $\simeq 200 \mathrm{yr}$ (corresponding to $\varpi_{b}$ 's precession period; $\varpi_{c}$ precesses much slower). The anti-correlated oscillations of inclinations relative to the transit plane are a geometrical effect resulting from the shared precession of $\Omega_{b}$ and $\Omega_{c}(\simeq 200 \mathrm{yr}$ period $)$. The inclinations relative to the invariant plane are nearly constant with the mutual inclination $\simeq 1^{\circ}$. If $I_{b}$ relative to the transit plane increases in the next decades (Fig. 3d), KOI-872b's transits will gradually disappear.

TTVs were originally proposed as a nontransiting planet detection method (2-4), but have recently found more use in validating the transiting planet candidates from Kepler $(24,25)$. Kepler has previously inferred the presence of a nontransiting planet via TTVs, but showed that the measurements were unable to support a unique solution (26). Here we have demonstrated the full potential of TTVs as a method to detect nontransiting planets and precisely characterize their properties.

\section{References and Notes}

1. J. A. Carter, J. C., Yee, J. Eastman, S. B. Gaudi, J. N. Winn, ApJ, 689, 499 (2008) 
2. J. Miralda-Escudé, ApJ, 564, 1019 (2002).

3. E. Agol, J. Steffen, R. Sari, W. Clarkson, MNRAS 359, 567 (2005).

4. M. J. Holman, N. W. Murray, Science, 307, 1288 (2005).

5. P. Sartoretti, J. Schneider, $A \& A S, \mathbf{1 3 4}, 553$ (1999).

6. D. M. Kipping, MNRAS, 392, 181 (2009a).

7. D. M. Kipping, MNRAS, 396, 1797 (2009b).

8. D. M. Kipping et al., ApJ, 750, 115 (2012).

9. W. J. Borucki et al., ApJ, 736, 19 (2011).

10. Materials and methods are available as supplementary material on Science Online.

11. F. Feroz, M. P. Hobson, MNRAS, 384, 449 (2008).

12. F. Feroz, M. P. Hobson, M. Bridges, MNRAS, 398, 1601 (2009).

13. M. Montalto, $A \& A, \mathbf{5 2 1}, 60$ (2010).

14. C. A. Scharf, ApJ, 661, 1218 (2007).

15. C. A. Watson \& T. R. Marsh, MNRAS, 405, 2037 (2010).

16. R. R. Rafikov, ApJ, 700, 965 (2009).

17. D. Nesvorný, C. Beaugé, ApJL , 709, L44 (2010).

18. D. Nesvorný, A. Morbidelli, ApJ, 688, 636 (2008).

19. We also tested the possibility of planets in the 1:1 mean motion resonance. We used an $N$ body integrator in this case, because perturbation theory described in (18) is not convergent for crossing orbits. 
20. M. J. Duncan, H. F. Levison, M. H. Lee, AJ, 116, 2067 (1998).

21. To demonstrate this we generated $10^{6}$ realizations in the neighborhood of $s 2$ and selected those consistent with the observed TTVs (as defined by $\chi^{2}<\chi_{\min , \mathrm{s} 2}^{2}+\delta \chi^{2}(99 \%)$ ). We found that the mass and orbit were well constrained from TTVs with $M_{c} \simeq 1.8 \times 10^{-3} M_{*}$, $81.5<P_{c}<82.3 \mathrm{~d}, 0.02<e_{c}<0.05$ and $7^{\circ}<I_{c}<12^{\circ}$. We then calculated TDVs for the selected realizations and found that all were inconsistent with the measurements by $>3 \sigma$. In contrast, the realizations near s 2 that were consistent with the observed TDVs had $I_{c}<5^{\circ}$, and were inconsistent with the observed TTVs.

22. We attempted to fit the observed TTVs with a three-planet system. The obtained solutions recovered the two-planet case corresponding to s1 or s2, with the third planet being either too small or too distant to produce significant TTVs.

23. J. J. Lissauer et al., ApJS 197, 8 (2011a).

24. M. J. Holman et al., Science 330, 51 (2010).

25. J. J. Lissauer et al., Nature 470, 53 (2011b).

26. S. Ballard et al., ApJ, 743, 200 (2011b).

27. T. M. Brown et al., ApJ, 552, 699 (2001).

This work is part of the Hunt for Exomoons with Kepler (HEK) project. We thank the Kepler Science Team, especially the DAWG, for making the data used here available. DN acknowledges support from NSF AST-1008890. DMK is supported by the NASA Sagan fellowship. GB acknowledges support from NASA grant NNX09AB29G. JH acknowledges support from NSF AST-1108686. 


\begin{tabular}{|c|c|c|c|}
\hline & $K O I-872 b$ & KOI-872c & KOI-872.03 \\
\hline$\tau_{0}\left[\mathrm{BJD}_{\mathrm{UTC}}\right]$ & $2455053.2826_{-0.0014}^{+0.0013}$ & - & $2455255.2603_{-0.0031}^{+0.0032}$ \\
\hline$P_{P}$ [days] & $33.60134_{-0.00020}^{+0.00021}$ & $57.011_{-0.061}^{+0.051}$ & $6.76671_{-0.00012}^{+0.00013}$ \\
\hline$R_{P} / R_{*}$ & $0.0887_{-0.0012}^{+0.0010}$ & - & $0.01656_{-0.00082}^{+0.00079}$ \\
\hline$b_{P}$ & $0.757_{-0.027}^{+0.022}$ & $2.9_{-1.8}^{+1.1}$ & $0.39_{-0.12}^{+0.19}$ \\
\hline$a_{P} / R_{*}$ & $45.1_{-1.7}^{+2.1}$ & $64.1_{-2.5}^{+2.9}$ & $15.58_{-0.49}^{+0.51}$ \\
\hline$i_{P}\left[^{\circ}\right]$ & $89.038_{-0.067}^{+0.075}$ & $87.4_{-1.0}^{+1.6}$ & $88.55_{-0.69}^{+0.49}$ \\
\hline$a_{P}[\mathrm{AU}]$ & $0.1968_{-0.0028}^{+0.0029}$ & $0.2799_{-0.0040}^{+0.0041}$ & $0.0679_{-0.0035}^{+0.0035}$ \\
\hline$e_{P}$ & $0.01_{-0.01}^{+0.01}$ & $0.0146_{-0.0036}^{+0.0034}$ & 0 (assumed) \\
\hline$\Omega_{P}\left[^{\circ}\right]$ & 270 & $298_{-36}^{+37}$ & - \\
\hline$\varpi_{P}\left[^{\circ}\right]$ & - & $330.0_{-9.2}^{+11.6}$ & - \\
\hline$\lambda_{P}\left[^{\circ}\right]$ & 0 & $338.2_{-1.4}^{+1.2}$ & - \\
\hline$M_{P} / M_{*}$ & $<6.4 \times 10^{-3}$ & $3.97_{-0.11}^{+0.15} \times 10^{-4}$ & - \\
\hline$M_{P}\left[M_{J}\right]$ & $<6$ & $0.376_{-0.019}^{+0.021}$ & - \\
\hline$R_{P}\left[R_{J}\right]$ & $0.808_{-0.043}^{+0.042}$ & - & $0.1510_{-0.0098}^{+0.0094}$ \\
\hline$\rho_{P}\left[\mathrm{~kg} \mathrm{~m}^{-3}\right]$ & $<14000$ & - & - \\
\hline$T_{\text {eq }}[\mathrm{K}]$ & $543_{-16}^{+16}$ & $455_{-14}^{+13}$ & $924_{-23}^{+24}$ \\
\hline \multirow[t]{2}{*}{$M_{\text {moon }} / M_{P}$} & $<0.021$ & - & - \\
\hline & KOI-872 & & \\
\hline$\rho_{*}\left[\mathrm{~kg} \mathrm{~m}^{-3}\right]$ & $1530_{-170}^{+220}$ & & \\
\hline$M_{*}\left[M_{\odot}\right]$ & $0.902_{-0.038}^{+0.040}$ & & \\
\hline$R_{*}\left[R_{\odot}\right]$ & $0.938_{-0.039}^{+0.038}$ & & \\
\hline $\log g_{*}$ & $4.447_{-0.035}^{+0.040}$ & & \\
\hline$T_{\text {eff }}[\mathrm{K}]$ & $5155 \pm 105$ & & \\
\hline$L_{*}\left[L_{\odot}\right]$ & $0.556_{-0.070}^{+0.078}$ & & \\
\hline$M_{V}$ & $5.60_{-0.17}^{+0.17}$ & & \\
\hline Age [Gyr] & $9.7_{-3.5}^{+3.7}$ & & \\
\hline Distance $[\mathrm{pc}]$ & $855_{-65}^{+68}$ & & \\
\hline$[\mathrm{M} / \mathrm{H}]$ & $0.41 \pm 0.10$ & & \\
\hline
\end{tabular}

Table 1: KOI-872 system parameters. KOI-872b parameters were computed from the weighted posteriors of a model accounting for TTVs, using MultiNeST. Parameters fitted in the transit model are quoted as the median of the marginalized posteriors with $\pm 34.13 \%$ credible intervals. Instrumental terms and times of transit minimum may be found in Table S3. KOI-872c parameters were computed from the fit to the KOI-872b's TTVs. Parameters fitted in the TTV model are quoted as maximum likelihood with $\pm 34.13 \%$ uncertainties computed using the $\Delta \chi^{2}=1$ method described in (27), where the TTV errors have been rescaled such that $\chi_{\text {reduced }}^{2}=1$. The $99 \%$ confidence areas from the TTV fit alone are shown in Fig. S10. The measured TDVs do not offer a meaningful constraint, since the parameter sets near s1 that fit the TTVs also fit the TDVs. Orbital longitudes of KOI-872c are relative to the transit reference system on 2455053.2839 BJD $\mathrm{UTC}_{\mathrm{UTC}}$ KOI-872.03 parameters were computed from an MCMC run. Moon mass constraint ( $3 \sigma$ limit) was derived from model $\mathcal{M}_{M T 2, R 0}(10)$. 


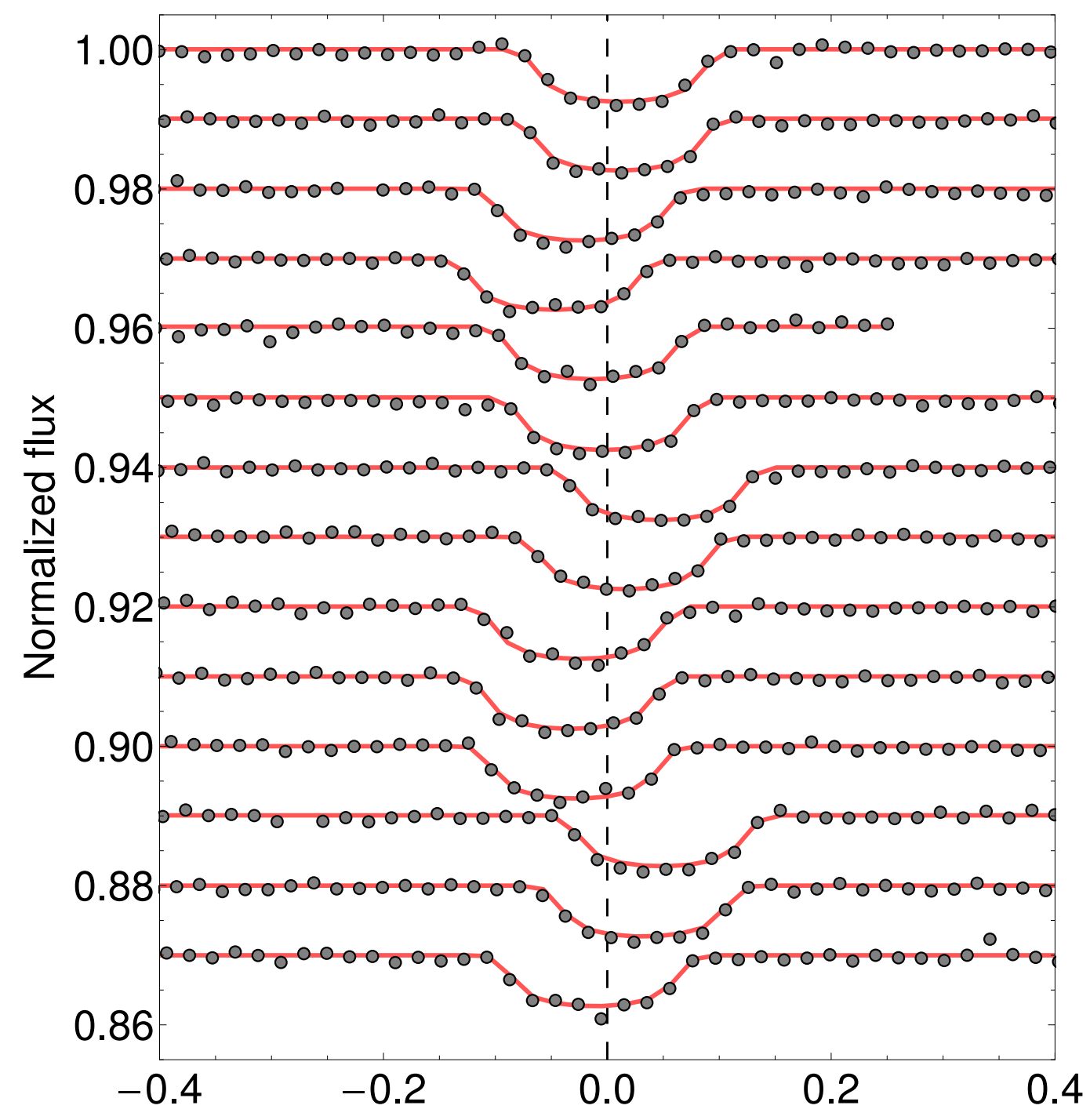

Days since transit minimum for a linear ephemeris

Figure 1: Maximum likelihood transit model (red line) overlaid with the long-cadence Kepler offsetted data for KOI-872b. The large TTVs are evident visually from the light curve. The ramp-affected transit is excluded here (see Fig. S2). 

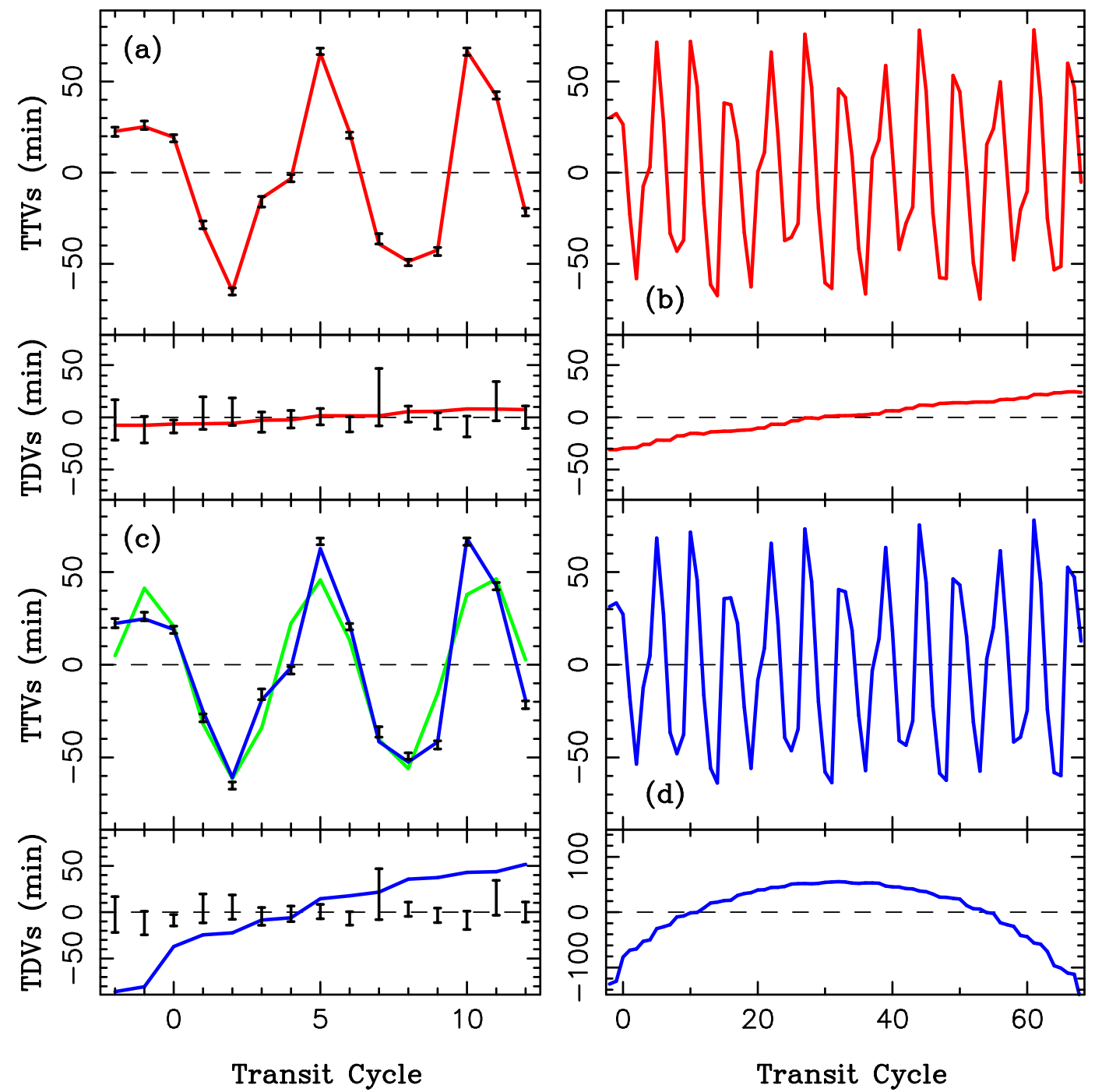

Figure 2: Transit timings variations. The measured TTVs (from model $\mathcal{M}_{T}$ ) and TDVs (from model $\mathcal{M}_{V}$ ) and their uncertainties are indicated in panels (a) and (c). Panel (a) shows the calculated values for s1 (red line). The TDVs of s1 are consistent with the measured, flat TDV profile. Panel (c) shows s2 (blue line) and our best moon model (green line). While s2 also fits the measured TTVs relatively well, the strong TDV trend in (c) is inconsistent with the measurements. Panels (b) and (d) show our predictions for s1 and s2. 

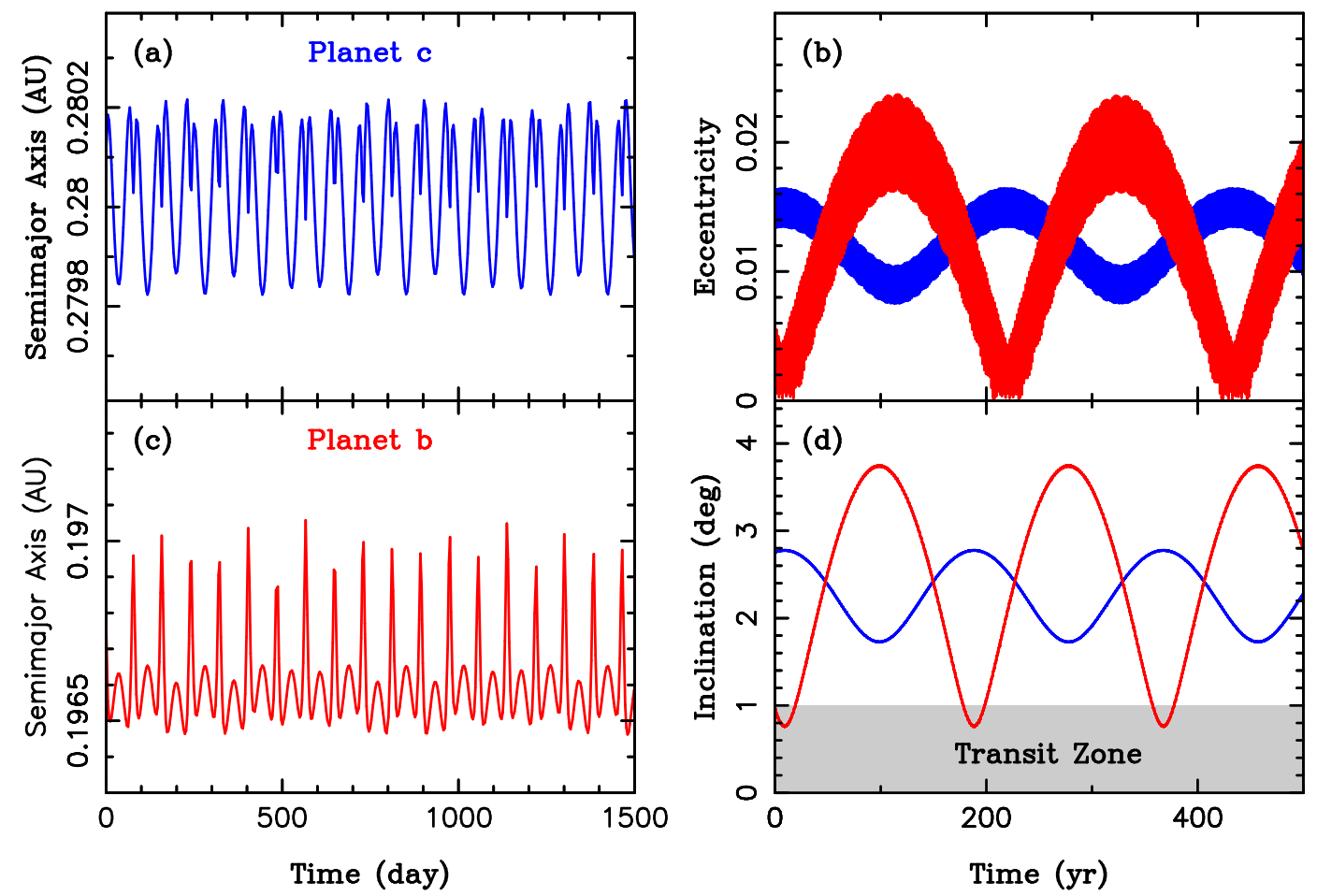

Figure 3: Orbit evolution. Figure shows the semi-major axis (panels a and c), eccentricity (b) and inclination $(\mathrm{d})$ for the best fit solution. The orbital elements of KOI-872b and KOI-872c are shown in red and blue, respectively. The inclination in (d) is defined relative to the transit plane ( $I=0^{\circ}$ would correspond to a transit across the middle of the host star's disk). An approximate transit zone for $\Omega=270^{\circ}$ is shaded. $\Omega_{b}$ and $\Omega_{c}$ are locked near $270^{\circ}$, because the invariant plane of planets is tilted to the transit plane. KOI-872.03 is omitted here because it has a negligible effect on the dynamical evolution of KOI-872b and KOI-872c. 


\section{Supporting Online Material}

System Identification We here discuss why KOI-872 was selected for analysis by the HEK project. The HEK project filters the list of all known Kepler transiting planet candidates down to a subset of the most promising for detecting exomoons. This process is described as "Target Selection" (TS) and features three distinct pathways: target selection visual (TSV), target selection automatic (TSA) and target selection opportunities (TSO). We direct the reader to (8) for details on each procedure.

KOI-872b, internally labeled as HCV/HCA-439.01, was identified independently by TSV and TSA as being an excellent candidate for exomoon follow-up and thus was prioritized for more detailed analysis. These determinations were initially based upon the Q1-Q3 long-cadence data only. Subsequent Q4-Q6 data is included in the analysis presented here.

Data Handling We will here describe the sequential steps we took in processing the Kepler photometry for the target KOI-872.

Data acquisition. We make use of the publicly available archival data from the Kepler Mission on the MAST website, which consists of quarters 1 through 6 (Q1-Q6). All data is in long-cadence mode with nearly continuous coverage. Full details on the data processing pipeline can be found in the data release handbooks and is not repeated here.

Long-term detrending with a cosine filter. We make use of the "raw" (labeled as "SAP_FLUX" in the header) data processed by the Kepler DAWG (Data Analysis Working Group) pipeline. All of the publicly available photometry was acquired in long-cadence (LC) mode spanning quarters 1 through 6 (Q1-Q6) and data releases 4 through 9 (DR4-DR9). A detailed description of the pipeline can be found in the accompanying release notes. The "raw" data has been processed using PA (Photometric Analysis), which includes cleaning of cosmic ray hits, Argabrightenings, removal of background flux, aperture photometry and computation of flux- 
weighted centroid positions.

The data releases also include corrected fluxes (labeled as "PDCSAP_FLUX" in the header), which are outputted from the PDC (Pre-search Data Conditioning) algorithm developed by the DAWG . As discussed in DR5, this data is not recommended for scientific use, owing to, in part, the potential for under/over-fitting of the systematic effects. For the sake of brevity, we do not reproduce the details of the PA and PDC steps here, but direct those interested to (28) and the DR handbooks.

The Q1 to Q6 PA photometry are shown in Fig.1(a), 1(b), 1(c), 1(d), 1(e) \& 1(f) respectively. One challenge in attempting a correction is assessing which components are astrophysical in nature and which are instrumental. The astrophysical signal of interest is the transit signal and thus we aim to detrend all other effects and preserve the eclipse. The spurious trends may be removed by applying a high-pass filter to the photometry, in a similar way as was used by (29) for CoRoT photometry and $(30,31)$ for Kepler photometry. To remove the long-term trends, we thus applied a discrete cosine transform (32) adopted to the unevenly spaced data.

We first removed the transit events with a margin of $\pm \mathfrak{T}$ days either side of the times of transit minimum, as predicted from the linear ephemeris period and epoch reported in (9). The choice of the time $\mathfrak{T}$ is provided later. We also remove outliers, identified as those points lying 3- $\sigma$ away from a spline-interpolated running median of window-size 600 minutes. Treating each quarter separately, we fitted the remaining data with a linear combination of the first $\mathrm{N}$ low-frequency cosine functions using

$$
f_{i}\left(t_{j}\right)=\cos \left[\left(\frac{2 \pi}{2 B}\right) t_{j} i+\phi_{i}\right],
$$

where $t_{j}$ is the timing of the $j^{\text {th }}$ measurement, $i=0, N$ in integer steps and $N$ is equal to the rounded integer value of $\left(2 B / 4 \mathfrak{T}^{\prime}\right)$ where $B$ is the timespan of the observations and $\mathfrak{T}^{\prime}$ is the timescale we are protecting. Since we are trying to protect the timescale $\mathfrak{T}$, we set $\mathfrak{T}^{\prime}=$ 
$3 \mathfrak{T}$ to ensure the timescale of interest in minimally distorted 1 . Using a Levenberg-Marquardt algorithm, we then fit for the linear coefficient, $q_{i}$, for each of the cosine functions, so that the fitted model is given by

$$
\mathfrak{M}\left(t_{j}\right)=\sum_{i=0}^{N} q_{i} f_{i}\left(t_{j}\right)
$$

We then subtracted model $\mathfrak{M}$ from the light curve (including the transits). The model is shown over the data for the Q1-Q6 photometry in Fig. 1(a), 1(b), 1(c), 1(d), 1(e) \& 1(f), The final time series is clipped to be $\pm 2 \mathfrak{T}$ surrounding the time of transit minimum, as based upon the linear ephemeris of (9).

Protected timescale. The harmonic filter protects a chosen timescale. When searching for ellipsoidal variations or reflected light, this timescale may be set to be equal to the period of the transiting planet, as was done in (29) and (33). However, if we are solely interested in the transit event, then the timescale of interest is much shorter than this. Specifically, it is the duration of the transit which is the timescale of interest. If we are looking for moons, then auxiliary eclipse signals may appear either side of the transit event. Thus, we wish to protect a timescale which includes both the transit duration and the surrounding temporal coverage corresponding to the region where a moon could reside. Spatially, this is the Hill radius and thus we need to define "a Hill timescale".

The first-to-fourth transit duration is already known from (9) to be $T_{14}=4.3863$ hours. If this time is roughly equal to the time is takes for the planet to traverse two stellar radii, $2 R_{*}$, then the velocity of the planet is given by $v_{P} \simeq 2 R_{*} / T_{14}$. The Hill timescale, $T_{H}$, is given by:

\footnotetext{
${ }^{1}$ For Q6, we were not able to get a good fit to the raw data using $\mathfrak{T}^{\prime}=3 \mathfrak{T}$ and so this factor was relaxed to unity.
} 


$$
\begin{aligned}
T_{H} & =\frac{R_{H}}{v_{P}}, \\
& \simeq \frac{a_{P} T_{14}}{2 R_{*}}\left(\frac{M_{P}}{3 M_{*}}\right)^{1 / 3} .
\end{aligned}
$$

where $R_{H}$ is the Hill radius, $a_{P}$ is the planet's semi-major axis, $M_{P}$ is the mass of the planet and $M_{*}$ is the mass of the star. In the above, $a_{P}, T_{14}, R_{*}$ and $M_{*}$ have reasonable estimates from (9). $M_{P}$, however, is wholely unknown. Since the planet is the Saturn-size regime, we simply adopt a Saturn-like density and estimate $M_{P}$ accordingly:

$$
T_{H} \simeq \frac{a_{P}}{2 R_{*}}\left(\frac{(4 / 3) \pi R_{P}^{3} \rho_{P}}{3 M_{*}}\right)^{1 / 3} .
$$

where $R_{P}$ and $\rho_{P}$ are the planet's radius and mean density respectively. For HCV/HCA439.01, we estimated $T_{H} \simeq 0.24$ days. The protected timescale, $\mathfrak{T}$, was then set to be equal to:

$$
\mathfrak{T}=1.2\left(T_{14}+2 T_{H}\right),
$$

where the 1.2 factor is added as a $20 \%$ buffer. In total, this gives $\mathfrak{T}=0.80$ days as the protected timescale.

Dilution factors. In each aperture, a small amount of third light is typically present from the overlapping point-spread-functions in Kepler's relatively crowded field. To account for this, it is necessary to include the third light in the transit light curve fits. We follow the method of (34) to make this correction. Although not available in the MAST headers directly, the dilution factors are accounted for in the PDC photometry but not in the PA photometry. Therefore, we simply take the median of both sets to compute the dilution factor. This is done for each quarter independently and ranged from $3 \%$ to $9 \%$. 
Light Curve Analysis Star spots. The raw transit light curve shows clear evidence for rotating star spots on the surface of KOI-872. In particular, Fig.1(d) shows three deep minima due to a large dark, rotating spot of roughly $10 \%$ the radius of the star. However, throughout the time series complex spot patterns emerge and evolve indicating the behavior is more complex than a simple, single spot model. For this reason, a simple periodogram is unable to reliably infer a rotation period. A more complex spot-model, accounting for multiple spots and differential rotation, is required to interpret the behavior of the these events, which is outside of the scope of this work.

For the purposes of this work, the most important consequence of the spots is that signals which appear like exomoon mutual-events may in fact be star spot crossings. Equipped with this prior information, a star spot crossing seems an a-priori more likely explanation for the TSV signals initially identified. These signals also complicate the derivation of upper limits on a putative exomoon, as discussed later. Upon downloading the Q4 data in early January, we immediately suspected the putative signal was a false-positive. However, we continued our analysis because our early model fits revealed that the planet exhibited large and complex transit timing variations, as also reported in $(35,36)$.

Model fits. In fitting the transit light curve, we consider a variety of models to explain the data. In all cases, the fitted parameter set is the same as that described in (8) with two exceptions. Firstly, rather than fitting for the satellite-to-planet mass ratio $\left(M_{S} / M_{P}\right)$ in planetwith-moon fits, we used $\left(\rho_{S}\right)^{2 / 3}$ i.e. mean density of the satellite to the power of two-thirds. This was done since a physical density has a better known prior than mass ratios. The two-thirds power ensures uniform priors in any derived mass-ratios since the other density terms feature this index for reasons described in (8). Secondly, we fit for a photometric noise term, $\sigma_{W}$, which is the standard deviation of the noise assuming it to be white. This is done to propagate the uncertainty of the photometric uncertainties themselves into our calculation of the Bayesian evidences. $\sigma_{W}$ is fitted with a modified Jeffrey's prior with the inflection point at the median 
photometric uncertainty from the DAWG pipeline and the maximum limit being 10 times larger. We note the best value for this term (from the $\mathcal{M}_{T}$ fits) is $\sigma_{W}=2.603_{-0.021}^{+0.022} \mathrm{mmag}$ per minute.

In what follows, we assume that the system is a genuine planetary system rather than a false-positive, such as a blended eclipsing binary. Details on our vetting procedure and blend analysis are provided later. The following principal models were considered:

- $\mathcal{M}_{P}$ - Planet-only

- $\mathcal{M}_{T}$ - Planet-only with variable times of transit minimum (TTV)

- $\mathcal{M}_{V}$ - Planet-only with variable times of transit minimum (TTV), transit depths (T $\left.\delta \mathrm{V}\right)$, impact parameters and $\left[\rho_{*}^{\text {circ }}\right]^{2 / 3}$ values 2 (thus permitting TDV)

- $\mathcal{M}_{M}$ - Planet-with-moon

- $\mathcal{M}_{M, R 0}$ - Planet-with-moon, defining the moon as a point-mass i.e. we fix $R_{S}=0$.

- $\mathcal{M}_{M T 1, M 0}$ - Planet-with-moon, removing the maximum a-posteriori transit timing variations deduced from $\mathcal{M}_{T}$. Since all TTVs are removed, we must enforce $M_{S}=0$.

- $\mathcal{M}_{M T 2, R 0}$ - Planet-with-moon, removing the maximum likelihood transit timing variations from a second planet fit and searching for residual TTVs/TDVs only.

The fits were executed using the MultiNest algorithm $(11,12)$, which is a multimodal nested sampling routine (37) designed to compute the Bayesian evidence in complex parameter space in an efficient manner. For brevity, we direct those interested to the aforementioned works for further details. MULTiNest is coupled with the forward-modeling code of LUNA (38), which is designed to model the transit light curves of a planet-with-moon accounting for mutual events, auxiliary transits, dynamical perturbations and non-linear limb darkening in an analytic manner.

\footnotetext{
${ }^{2} \rho_{*}^{\text {circ }}$ is the light curve derived stellar density assuming a circular orbit.
} 
Quadratic limb darkening coefficients were estimated in a similar manner to that described in (30). For this calculation, we assumed the effective temperature and surface gravity of the star to be that reported in (9) $\left(T_{\text {eff }}=5127 \mathrm{~K}\right.$ and $\left.\log g_{*}=4.59\right)$, which in turn come from the Kepler Input Catalogue (KIC). Our later spectroscopic analysis shows these to be excellent estimates. For the Kepler bandpass, we used the high resolution Kepler transmission function found at http://keplergo.arc.nasa.gov/CalibrationResponse.shtml We employed the atmosphere model database from (39) providing intensities at 17 emergent angles, which we interpolated linearly at the adopted $T_{\text {eff }}$ and $\log g_{*}$ values. The passband-convolved intensities at each of the emergent angles were calculated following the procedure in (40). This whole process is performed by a Fortran code written by I. Ribas. To compute the coefficients we used the limb darkening law given in Equation 6 ,

$$
\frac{I_{\mu}}{I_{1}}=1-u_{1}(1-\mu)-u_{2}(1-\mu)^{2},
$$

where the various terms are defined in (40). The final coefficients resulted from a least squares singular value decomposition fit to 11 of the 17 available emergent angles. The reason to eliminate 6 of the angles is avoiding excessive weight on the stellar limb by using a uniform sampling (10 $\mu$ values from 0.1 to 1 , plus $\mu=0.05$ ), as suggested by (41). This leaves us with $u_{1}=0.3542$ and $u_{2}=0.3607$.

Ramp correction. During the Kepler time series, there are a few safe mode events where the telescope stopped observing. These result in a pause in the continuous photometry followed by an exponential ramp as the telescope starts observing again. This ramp, likely similar to the charge trapping effect seen with Spitzer (42), lasts for around one week and we usually simply clip the affected data. Unfortunately, the second transit observed occurs during one these ramps. In order to maximize the available data, we decided to correct for the ramp effect and recover this transit. We apply a simple exponential decay model of the form 


$$
F^{\prime} / F=a_{0}-a_{1} \exp \left(-t / a_{2}\right),
$$

where $F^{\prime}$ is the flux affected by the ramp, $F$ is the flux corrected for the ramp and $t$ is the time since the start of the ramp. We also tried a double exponential, similar that advocated by (42), but found the two timescales converged to a single value. In fitting the data, we fit the parameters $a_{0}$ (absorbed by the OOT vector in practice), $a_{1}$ and $a_{2}$ simultaneously to fitting the transit model. We find that this simple model provides an excellent description of the ramp effect, as visible in Fig \$2 , We note that the ramp timescale was found to be best modeled by $a_{2}=1.1024_{-0.0069}^{+0.0069}$ days (from $\mathcal{M}_{T}$ fits), which may be useful to other observers.

Model selection. In searching for an exomoon, one must conduct model selection between the various hypotheses which could explain the data. We define our null model to be that of a transiting planet without a moon and with static parameters; model $\mathcal{M}_{P}$. This simply assumes a constant linear ephemeris with a constant duration and depth every transit.

We also consider models of a planet without a moon, but with perturbations. The simplest type of perturbation model we consider is that of a planet with varying times of transit minimum (i.e. a planet experiencing TTV) which we dub $\mathcal{M}_{T}$. Model $\mathcal{M}_{V}$ extends this to allow for variable depth and duration as well. Models $\mathcal{M}_{M}$ and $\mathcal{M}_{M, R 0}$ are the planet-with-moon model, as simulated from LUNA, except the latter assumes a fixed zero-radius moon $\left(R_{S}=0\right)$; i.e. considers TTVs and TDVs only.

Due to the presence of a second planet inducing the TTVs (as discussed in the main text), we also tried models $\mathcal{M}_{M T 1, M 0}$ and $\mathcal{M}_{M T 2, R 0}$. The first model removes all transit timing variations by subtracting the maximum a-posteriori transit times deduced using $\mathcal{M}_{T}$ from the original data and then refitting for a zero-mass exomoon (we cannot fit for a moon mass if we have forcibly removed all TTVs). The second subtracts the maximum likelihood model for a second planet (discussed later) causing the TTVs and then fits the adjusted data for a planet-with-moon. In 
this case, we also fix the moon radius to be zero since we later hypothesize that stellar activity may be inducing false-positive moon-like eclipses. Therefore, this model looks for residual TTVs and TDVs only. Because these fits use modified data for inputs, they cannot be directly compared to the other models and require a custom null-model for comparison in each case.

Model selection is performed by comparing the Bayesian evidence of each model. The higher the Bayesian evidence the more likely the model is the correct one. Computing the Bayesian evidence is computationally expensive and particularly challenging in the high-dimensional space we are faced with. For example, $\mathcal{M}_{T}$ involves 38 free parameters.

In some cases, we found it was not necessary or possible to formally compute the absolute value of the Bayesian evidence, $\mathcal{Z}$. For example, as MultiNEST iteratively searches through higher $\log \mathcal{Z}$ values, the code can be stopped if the $\log \mathcal{Z}$ value greatly exceeds the evidence of a competing hypotheses. This allows us to place a lower limit on the confidence of such a model, as was done for $\mathcal{M}_{T}$. Here, we found $\left(\log \mathcal{Z}_{T}-\log \mathcal{Z}_{V}\right) \geq(166.1 \pm 0.7)$ (where model $\mathcal{M}_{V}$ has the next-best Bayesian evidence), indicating $\geq 18.1-\sigma$ preference for the TTV model over the TTV+TDV+T $\delta \mathrm{V}$ model. To highlight the computational demands, we point out that even this lower limit required over 3 years of equivalent processing time, including over 4 billion likelihood evaluations, with a $2.1 \mathrm{GHz}$ AMD Interlagos CPU. We also note that the TTV model is preferred over the static model, $\mathcal{M}_{P}$, at a confidence of $\geq 43.9-\sigma$, which to our knowledge represents the highest formal significance for a TTV detection ever reported. For cases where only a lower limit on $\mathcal{Z}$ is provided, the posteriors were computed by re-running MULTINEST in constant efficiency mode.

Models $\mathcal{M}_{M}$ and $\mathcal{M}_{M, R 0}$ are found to be relatively poor fits to the data with $\left(\log \mathcal{Z}_{M}-\right.$ $\left.\log \mathcal{Z}_{T}\right) \leq-(635.3 \pm 0.4)$ and $\left(\log \mathcal{Z}_{M, R 0}-\log \mathcal{Z}_{T}\right) \leq-(545.8 \pm 0.4)$. This is visually evident by comparing the TTV model for a second planet versus that of a moon in Fig. 1. Since $\mathcal{Z}_{T} \gg \mathcal{Z}_{P}$, there is a very high probability that transit timing variations are present. But since a moon model provides a much lower evidence than the TTV model (i.e. $\mathcal{Z}_{M} \ll \mathcal{Z}_{T}$ ), then we 
deduce that i) TTVs are present ii) a moon is not responsible.

Although this conclusion tells us the large TTVs are not being caused by a moon, they do not exclude the presence of a moon either. The possibility of a moon as an independent source of TTVs is investigated using models $\mathcal{M}_{M T 1, M 0}$ and $\mathcal{M}_{M T 2, R 0}$. We did not directly compare the Bayesian evidence for these models with the others. This is because the input data was manipulated in each case by subtracting times of transit minimum from each epoch. In order to perform a reliable model comparison, we generated a custom null model for each: $\mathcal{M}_{M T 1 \text {,null }}$ and $\mathcal{M}_{M T 2, \text { null }}$. These were accomplished by re-running the fit with the size and mass of the moon set to zero, allowing us to remove the moon terms as free parameters. The results of these fits will be discussed later in a dedicated section.

The priors and Bayesian evidence values for each model we attempted are provided in Tables $\mathbf{S 1} \& \mathbf{S 2}$ respectively. Table 1 provides the final physical system parameters. Table $\mathbf{S 3}$ provides the final system parameters for instrumental terms and times of transit minimum.

A Search for an Occultation Searching for an occultation is challenging due to the large transit timing variations present. In order to accommodate for this, we allow the occultations to have their own timing variations. To minimize the number of free parameters, the $p, b_{P}$, $\left[\rho_{*}^{\text {circ }}\right]^{2 / 3}, P_{P}$ and $\tau_{0}$ parameters were sampled from a Gaussian prior derived from the $\mathcal{M}_{T}$ model fit. Since any eccentricity induced timing offsets should be absorbed by the occultation timing variations, fitting for eccentricity terms would be essentially fitting redundant parameters and thus we fix $e_{P}=0$. This left us with 15 OOT parameters, 15 occultation times, 1 noise parameter $\left(\sigma_{W}\right)$ and 1 term for $\left(\mathcal{F}_{P} / \mathcal{F}_{*}\right)$, the flux-per-unit-area ratio of the planet and star. We ran two fits; one where $\left(\mathcal{F}_{P} / \mathcal{F}_{*}\right)=0$ (in such a case the occultation times are not required as free parameters) and one where $0<\left(\mathcal{F}_{P} / \mathcal{F}_{*}\right)<1$ and was a uniform prior, allowing us to perform a model comparison later. Note that under the assumption that the system is a real planetary system, the TTVs suggest $e_{P} \simeq 0$ anyway, as described in the main text. 
A comparison of the Bayesian evidence from the null fit versus the occultation fit yields $\left(\log \mathcal{Z}_{\text {occ }}-\log \mathcal{Z}_{\text {null }}\right)=(-2.43 \pm 0.44)$. Thus, the null hypothesis of there being no occultation present is the preferred model.

Although no occultation is detected, we may use our results to place upper limits on the occultation depth. The marginalized posterior of of the occultation depth yields $\delta_{\text {occ }}=9.9_{-7.4}^{+14.9} \mathrm{ppm}$, and places a $3-\sigma$ upper limit of $\delta_{\text {occ }}<71.0 \mathrm{ppm}$. Assuming a geometric albedo of unity $\left(A_{g}=1\right)$, the reflected light component of the occultation is expected to be $3.9 \mathrm{ppm}$. Assuming the occultation is due to reflected light only, our occultation depth limit corresponds to $A_{g}<18.0$ i.e. we are unable to constrain the albedo of KOI-872.01 to any physically plausible range.

Given our insensitivity to reflected light, our upper limit is more robust and meaningful when interpreted as an upper limit on thermal emission. Kepler's visible bandpass is not wellsuited to detecting thermal emission but a meaningful constraint can still be derived. Treating the star and planet as black bodies and integrating over the custom Kepler bandpass, we find $T_{P}<2442 \mathrm{~K}$ to $3-\sigma$ confidence (assuming $T_{*}=5155 \mathrm{~K}$ ).

Vetting the Planetary System So far, we have assumed the observations are due to an unblended planet transiting a star. Here, we consider alternative models which do not require the system to include a planet. To avoid confusion with the earlier model fits which assume the eclipsing object is an unblended planet, we will dub these models as hypotheses, $\mathcal{H}_{i}$. There at least three such hypotheses which could potentially explain the data:

- $\mathcal{H}_{P}$ : No blend is present and thus we have a planet transiting a star

- $\mathcal{H}_{E B, 33.6}$ : A blended eclipsing binary (EB) with the eclipsing bodies on an orbital period of $33.6 \mathrm{~d}$

- $\mathcal{H}_{E B, 67.2}$ : A blended eclipsing binary (EB) with the eclipsing bodies on an orbital period 
of $67.2 \mathrm{~d}$

In the last two cases, the eclipsing binary could be a larger planet (e.g. Jupiter-sized) eclipsing a star and thus could still be considered a genuine planetary system. Further, the blend source could be foreground, background, associated or a mixture of multiple sources. The last two cases can also be considered in two flavors i) EB on a circular orbit ii) EB on an eccentric orbit. The possibility of an unblended grazing eclipsing binary is included in the model $\mathcal{H}_{P}$, and a blended grazing eclipsing binary within $\mathcal{H}_{E B, 33.6}$ and $\mathcal{H}_{E B, 67.2}$.

In general, one expects the false-positive rate for Kepler Objects of Interest to be quite low, with recent estimates arriving at $\lesssim 10 \%$ (43). Nevertheless, we will here investigate the possibility of the blended EB scenarios mimicking a planetary system. We will approach this problem using several tools 1) a centroid analysis 2) constraints from the spectroscopy 3) model selection with Bayesian evidence determinations of the transit light curve shape (a blend analysis) 4) dynamical constraints from the timing variations and stability arguments.

A Centroid Analysis Overview. The DAWG pipeline output provides flux-weighted centroid positions for all observed targets. (44) have demonstrated that the very small shifts in centroid, expected for blended occulting sources, can be measured accurately from the Kepler data. Consider two physically separated sources with overlapping PSFs. The computed centroid position is flux-weighted with respect to these two sources. When one of the sources is eclipsed, its flux temporally decreases and thus the flux-weighted centroid shifts towards the other source. Therefore, the detection of a shift in flux-weighted centroids during the eclipses would indicate the presence of a blend source. An example of this technique detecting such a source is for KOI-13 (45).

Excluded Centroid Shift. We extracted the $x$ and $y$ centroid positions for KOI-872 surrounding $\pm 0.4 \mathrm{~d}$ of the predicted transit events according to the linear ephemeris model derived in $\mathcal{M}_{P}$. We then removed the maximum a-posteriori times of transit minimum, $\tau$, for each transit 
epoch, as computed by the $\mathcal{M}_{T}$ model. This step essentially phases all of the data. Finally, we then divided epoch by the median $x$ and $y$ centroid position to remove the effect of long-term trends in the centroid positions. No cleaning or detrending of the centroids was attempted.

The phased centroid positions, shown in Fig. S3, display no obvious up or down pattern during the transit events (marked by the vertical gridlines). The scatter can be seen to be comparable, but somewhat larger than, that derived for KOI-13 in Fig. 2 of (45). Taking the mean and standard deviation of the in- versus out-of-transit centroid positions we find:

$$
\begin{aligned}
& \Delta x=x_{\text {out }}-x_{\text {in }}=(0.09 \pm 0.57) \times 10^{-4}, \\
& \Delta y=y_{\text {out }}-y_{\text {in }}=-(0.07 \pm 0.58) \times 10^{-4} .
\end{aligned}
$$

Defining $\Delta r=\sqrt{\Delta x^{2}+\Delta y^{2}}$, we determine $\Delta r=(0.12 \pm 0.57) \times 10^{-4}$, where all units thus far have been given in units of pixel position. This analysis clearly indicates that the data are consistent with no separated blend source present. The 3- $\sigma$ upper limit, converted to arcseconds, corresponds to $\Delta r<0.68$ mas, demonstrating the impressive performance of Kepler once again.

Excluded Blends. We here describe a toy model to interpret this upper limit. We consider two sources of flux $F_{*}$ and $F_{B}$ (star and blend source), where the star is transited. The fluxweighted positions, $r$, of the centroid in- and out-of-transit are given by:

$$
\begin{aligned}
r_{\text {out }} & =\frac{r_{*} F_{*}+r_{B} F_{B}}{F_{*}+F_{B}}, \\
r_{\text {in }} & =\frac{r_{*} F_{*}(1-\delta)+r_{B} F_{B}}{F_{*}(1-\delta)+F_{B}},
\end{aligned}
$$

where $\delta$ is the unblended eclipse depth. Since we only detect the blended eclipse depth, $\delta_{\mathrm{obs}}$, we must convert between the two using the expression from (34): 


$$
\delta=\delta_{\mathrm{obs}}(1+\beta)
$$

where $\beta=F_{B} / F_{*}$. If we also make the replacement $r_{B}=r_{*}+\Delta r$, one can write:

$$
\begin{aligned}
\Delta r_{\mathrm{obs}} & =r_{\mathrm{in}}-r_{\mathrm{out}}, \\
& =\frac{\beta \delta_{\mathrm{obs}} \Delta r}{(1+\beta)\left(1-\delta_{\mathrm{obs}}\right)} .
\end{aligned}
$$

Here, $\Delta r_{\text {obs }}$ represents the observed change in centroid position during the eclipse, in arcseconds, and $\Delta r$ represents the physical separation of the two sources on the sky, in arcseconds. Defining the blend factor as $B=(1+\beta)$, one may solve inverse the above expression to solve for $B$, as a function of $\Delta r$ :

$$
B(\Delta r)=\frac{\delta_{\text {obs }} \Delta r}{\delta_{\text {obs }} \Delta r-\Delta r_{\text {obs }}+\delta_{\text {obs }} \Delta r_{\text {obs }}}
$$

Using this simple model, we plot the excluded values of $B$ as a function of $\Delta r$ in Fig. S4, Note that this result is purely based on the centroid shifts. To quote several values from the figure, $B<1.239$ for $\Delta r<0.5^{\prime \prime}, B<1.051$ for $\Delta r<2^{\prime \prime}$ and $B<1.016$ for $\Delta r<6^{\prime \prime}$. A blend factor of $\simeq 10 \%$ does not impact significantly on our results and thus only blends within $\Delta r<0.5^{\prime \prime}$ could possibly cause a planet false-positive. We note that, in general, such a closelyspace companion is quite rare, with a recent adaptive optics campaign on suitable KOIs finding only $6.7 \%$ of KOIs have a companion within $0.5 \%$.

Fig. S4 also provides the same constraints as computed for the inner transiting planet candidate KOI-872.03 (dashed line). Details on the detection of this candidate are provided later. Due to the much smaller depth, the upper limits are less constraining but nevertheless still consistent with the absence of a blend source. 
A Spectroscopic Analysis. Observations. Spectroscopic observations of the star HCV/HCA439 were carried out with the Astrophysical Research Consortium Echelle Spectrograph (ARCES) on the Apache Point Observatory $3.5 \mathrm{~m}$ telescope located at Apache Point Observatory (APO) in New Mexico. We used a 1". $6 \times 3$ "'.2 slit which delivers a spectral resolution of $\Delta \lambda / \lambda \approx 31,000$ over a spectral range of $3200-10,000 \AA$. We obtained a total of two thirty minutes exposures on the star HCV/HCA-439 on the night of 2012 Jan 31 which, when combined, yielded a total S/N of 11 at $5100 \AA$. After performing a standard overscan correction, we removed cosmic rays, extracted the spectra, applied a flat-field correction, and determined the dispersion correction from a ThAr lamp spectrum using standard techniques in the IRAF's IMRED, CRUTIL, and ECHELLE packages.

Stellar Parameters. We used the Yonsei-Yale (YY) isochrones (46) to determine the physical properties of the host star. The first input into this analysis were the stellar atmosphere parameters determined from the APO $3.5 \mathrm{~m}$ spectra. We used the Stellar Parameter Classification (SPC) method to derive the stellar atmosphere parameters. SPC cross-correlates the observed spectrum against a grid of synthetic spectra drawn from a library calculated by John Laird using Kurucz models (47). The synthetic spectra cover a window of $300 \AA$ centered near the gravity-sensitive $\mathrm{Mgb}$ features and has a spacing of $250 \mathrm{~K}$ in effective temperature, $0.5 \mathrm{dex}$ in gravity, 0.5 dex in metallicity and $1 \mathrm{~km} \mathrm{~s}^{-1}$ in rotational velocity. To derive the precise stellar parameters between the grid points, the normalized cross-correlation peaks were fitted with a three dimensional polynomial as a function of effective temperature, surface gravity and metallicity. This procedure was carried out for different rotational velocities and the final stellar parameters were determined by a weighted mean of the values from the spectral orders covered by the library.

The isochrone analysis made used of the stellar effective temperature $T_{\text {eff }}=(5155 \pm 105) K$, and the metallicity $[\mathrm{Fe} / \mathrm{H}]=(0.41 \pm 0.10)$ from the SPC analysis. The second input into the YY analysis came from the light curve analysis of the Kepler data. The transit duration is closely 
related to the $a_{P} / R_{*}$ parameter, which in turn determines the mean stellar density $\rho_{*}(48)$. In general, this trick is only possible for systems where the orbital eccentricity is precisely known (49). It will be shown in the dynamical analysis discussion later that planet b's eccentricity is indeed strongly constrained from the TTVs to be near-circular. We proceed by using the light curve derived stellar density posterior from our model fits, giving $\rho_{*}=1520_{-170}^{+220} \mathrm{~kg} \mathrm{~m}^{-3}$.

The mean stellar density acts as a luminosity indicator for the star; smaller density typically means more evolved stars. Another possible luminosity indicator would be the surface gravity of the star, as determined from the spectroscopic analysis. If the eccentricity of the system is well determined and the light curve is of high quality (both of which are true here), then $a_{P} / R_{*}$ and the corresponding $\rho_{*}$ is typically a better luminosity indicator than $\log g_{*}$, in the sense that the derived physical parameters have a smaller error. We have generated over 10,000 values of $T_{\text {eff }},[\mathrm{Fe} / \mathrm{H}]$ and $a_{P} / R_{*}$ using their a posteriori distribution (assuming Gaussian distribution for the first two), and searched the YY isochrones for each materialization. About $95 \%$ of the input values had a matching isochrone. Stellar parameters were then determined as the median of the resulting distribution. The final parameters were $M_{*}=0.90 \pm 0.04 M_{\odot}, R_{*}=0.94 \pm 0.04 R_{\odot}$, and $\log g_{*}=4.44 \pm 0.04$ (cgs). The isochrones and the final solution are shown in Fig. S5, where the backdrop of isochrones is for ages $0.2,0.5,1.0,2.0, \ldots 13.0 \mathrm{Gyr}$ (from bottom to top) and $[\mathrm{Fe} / \mathrm{H}]=0.41$. The solution indicates an old star with $10 \pm 3 \mathrm{Gyr}$ age.

In regard to vetting of the system, we find no evidence for double-lines indicating a blend. We also find the spectral classification of the star very well described as a dwarf main-sequence star, and exclude the possibilities such as a giant star or white dwarf. We note that our classification is in close agreement with the KIC determination reported in (9).

A Blend Analysis Overview. The third vetting tool we use is model selection with Bayesian evidence determinations of the transit light curve shape i.e. a blend analysis. Blend analyses have become a powerful instrument in the toolbox of the Kepler team too, using their 
custom BLENDER software $(50,51)$. Here, the team simulate a grid of billions of possible false-positive scenarios and compute the odds ratio of valid planet solutions versus valid falsepositive solutions. Systems strongly favoring the planet solution are considered to be "validated". BLENDER makes use of additional information such as centroid positioning, priors of the frequency of eclipsing binaries and multi-color light curves (often from Spitzer (51)). In this work, we limit our analysis to purely an inspection of the shape of the light curve. This is done to reduce the computational demands of simulating billions of false-positives, and yet take advantage of the fact we have other more powerful constraints from the dynamics (as discussed later). Further, a centroid analysis has already indicated that a blend must be within 0.5 " to have a significant impact on our results and thus can be treated as a separate line of evidence.

Although our blend analysis is more simplified than BLENDER, it does take advantage of the Bayesian evidence for model selection, which is currently not implemented in BLENDER or other blend analyses in the current literature. Our approach is to consider a null hypothesis and then more elaborate models involving blend scenarios and compute the Bayesian evidence of each model. Since the more elaborate models include more parameters (i.e. a greater prior volume), they are penalized in the computation of the Bayesian evidence. If a blend model provides a Bayesian evidence significantly greater than that of the null model $(\Delta \log \mathcal{Z} \gtrsim 5)$, it passes the first test to becoming the preferred model. The second test we impose is that the parameter posteriors of the model must correspond to a physically plausible scenario. If both of these criteria are satisfied, then the null model can be displaced.

Another possibility is that two or more hypotheses yield approximately equal Bayesian evidences, such that there is no statistically significant preference between them. In such a case, we continue to consider these hypotheses as plausible descriptions of the system and test whether they are consistent with our subsequent dynamical analysis too.

The null hypothesis is the simplest model which can explain the data and so involves the fewest parameters to describe the system. In our case, this represents just two eclipsing objects 
without a blend. Whilst an unblended grazing eclipsing binary could fall into this category, it will be shown shortly that such a hypothesis is highly improbable. Therefore, the null hypothesis essentially represents a planet-sized object transiting a star.

Implementation. In our blend analysis, we are only investigating the shape of the light curve. The dynamical constraints from the timing variations will be discussed later, and so here we eliminate them by subtracting any TTVs away from a linear ephemeris. This is accomplished using the maximum a-posteriori transit times from the model fit $\mathcal{M}_{T}$ performed earlier. This reduces the number of free parameters by 15 and makes the fits far easier to handle computationally. However, we still fit each eclipse epoch with a unique baseline to remove any residual DC power from the detrending procedure.

For the null hypothesis, $\mathcal{H}_{\mathrm{P}}$, we have 15 OOT baseline parameters, $p, b_{P},\left[\rho_{*}^{\text {circ }}\right]^{2 / 3}, P_{P}, \tau_{0}, 2$ instrumental terms to describe the ramp effect for the one affected event and two limb darkening parameters $\left(u_{1}\right.$ and $\left.\left(u_{1}+u_{2}\right)\right)$, giving 24 parameters in total. Limb darkening was fitted for to provide a fair comparison to the blend models where limb darkening cannot be assumed to be the same as the theoretical models used in the planetary fits. The priors on the limb darkening terms were selected such that the brightness profile is positive everywhere and monotonically decreasing from limb to center, specifically $0<u_{1}<2$ and $0<\left(u_{1}+u_{2}\right)<1(52)$.

We also extended the prior on $p$ to the range $0<p<1$ and similarly for the impact parameter $0<b_{P}<2$. The other fitted terms had the same priors as used before (see Table S1).

For the $33.6 \mathrm{~d}$ period EB scenarios, we simply add a single additional term, a blending factor $B$. Our model for the diluted light curve follows the prescription of (34), where $B=1$ indicates no blend and $B>1$ indicates a blend. The model is general in that the source of the blend could be foreground, background, associated or a mixture. The prior on the blending factor was chosen to be $1<B<100$, and all other priors were left unchanged from $\mathcal{H}_{P}$. In general, one expects the $33.6 \mathrm{~d}$ blended EB scenario to give an equally good fit to the data as a planet, since the $B$ term is degenerate with the other fitting parameters (34). For the eccentric 
cases, we fitted for $e_{P}$ and $\omega_{P}$ directly using $0<\omega_{P}<2 \pi$ and $0<e_{P}<0.9$ uniform priors. The eccentricity was cut-off from extremely eccentric orbits to save CPU time, since solving Kepler's equation takes dramatically longer in the extreme eccentricity regime.

The final scenario of a $67.2 \mathrm{~d}$ period blended EB was treated by first modifying the prior on the period to be uniform around \pm 1 day of $67.2 \mathrm{~d}$. Then, we instructed the code to treat the occultations has having zero-limb darkening. Whilst the ingress/egress of the occultation may in fact have limb darkening, the 2nd-to-3rd contact of the transit, which dominates the signalto-noise, cannot have a limb darkened profile. Therefore this approximation contains the most important physics of the problem. The primary transit is treated as a limb darkened event as before. The occultation depth is equal to $p^{2}\left(\mathcal{F}_{P} / \mathcal{F}_{*}\right)$, where $\mathcal{F}$ is the flux-per-unit-area of a body. This flux ratio is the only new parameter required, for which we use $0<\left(\mathcal{F}_{P} / \mathcal{F}_{*}\right)<2$ as our prior. In general, one expects this scenario to be easier to distinguish against a planet due to the lack of curvature in alternate eclipses.

Results: An Unblended Grazing EB. The easiest scenario to disregard is that of an unblended grazing eclipsing binary. Such a scenario would be permitted in model $\mathcal{H}_{P}$ and may be tested for by evaluating the number of posterior samples which satisfy $b_{P}>(1-p)$; the definition of a grazing event. We find that $\left(b_{P}+p\right)<1$ to $>99.99 \%$ confidence and not a single posterior sample landed in this regime. Therefore, the hypothesis of an unblended grazing EB is highly improbable.

Results: A Blended Grazing EB. A blended grazing eclipsing binary could feature in all four of the alternative hypotheses; $\mathcal{H}_{E B, 33.6}^{c}, \mathcal{H}_{E B, 33.6}^{e}, \mathcal{H}_{E B, 67.2}^{c} \& \mathcal{H}_{E B, 67.2}^{e}$. The first thing to note is that Bayesian evidence of all of these models is not significantly improved over the null hypothesis, $\mathcal{H}_{P}$. In each of the four models, we checked the posterior samples satisfying a non-grazing configuration and found $\left(b_{P}+p\right)<1$ to $>99.99 \%$ in all four. Therefore, the hypothesis of a blended grazing EB is highly improbable.

Results: A Blended $67.2 \mathrm{~d}$ EB. As discussed earlier, the $67.2 \mathrm{~d}$ period blended EB causes 
a limb darkened transit but a flat-bottomed occultation. This difference in transit profile is expected to make the scenario easier to distinguish than the $33.6 \mathrm{~d}$ blended EB. Indeed, this is what we found. Hypotheses $\mathcal{H}_{E B, 67.2}^{c}$ and $\mathcal{H}_{E B, 67.2}^{e}$ yield $\Delta \log \mathcal{Z}=(-22.7 \pm 0.5)$ and $(-133.1 \pm 0.5)$ respectively, relative to the null hypothesis $\mathcal{H}_{P}$. This constitutes a $6.4-\sigma$ and $16.1-\sigma$ preference for the null hypothesis, for the two alternative hypotheses respectively. Therefore, the hypothesis of a blended $67.2 \mathrm{~d}$ EB is highly improbable.

Results: A Blended 33.6 d Circular Orbit EB. With all previous scenarios now rejected, we are left with the blended $33.6 \mathrm{~d}$ EB only, which comes into two flavors: $\mathcal{H}_{E B, 33.6}^{c} \& \mathcal{H}_{E B, 33.6}^{e}$. Let us here consider the circular case first. Strictly considering the Bayesian evidence results of our light curve profile analysis, there is no significant preference between the hypothesis of an unblended planetary transit and a blended $33.6 \mathrm{~d}$ EB. Note that these remaining blend scenarios include a Jupiter-sized planet transiting a blended main-sequence star.

Scenarios involving a giant star or white dwarf can be excluded based upon the spectroscopic analysis discussed earlier. The ratio-of-radii in both $\mathcal{H}_{E B, 33.6}^{c}$ is constrained to be $p<0.30$ to $>99.99 \%$. This means we must be dealing with a main-sequence star being transmitted by a smaller object, albeit with the possible presence of a blend.

We discussed earlier how there is no detectable occultation in the data between the $33.6 \mathrm{~d}$ transits. Under the hypothesis of $\mathcal{H}_{E B, 33.6}^{c}$, the other object must have $\left(\mathcal{F}_{P} / \mathcal{F}_{*}\right)<0.0089$ to 3- $\sigma$ confidence. This indicates a small $(p<0.3)$, cool $(T \lesssim 3000 \mathrm{~K})$ object consistent with a planet or brown dwarf.

Results: A Blended 33.6d Eccentric Orbit EB. The final case of the eccentric $33.6 \mathrm{~d}$ EB yields a slightly improved Bayesian evidence over the planet-only model, with a significance of $(2.4 \pm 0.2)-\sigma$. We do not consider this significant enough to overturn the planet hypothesis but nevertheless the scenario is considered plausible at this stage. A possible reason for the slight improvement is the ability of an eccentric orbit to generate a more diverse range of limb darkening profiles than the circular orbit case, when both models utilize quadratic limb 
darkening.

Results: Conclusions. We conclude that the only models which can adequately explain the spectroscopic analysis and the blend analysis are that of a planet transiting a star $\left(\mathcal{H}_{P}\right)$, a blended 33.6 d EB on a circular orbit $\left(\mathcal{H}_{E B, 33.6}^{c}\right)$ and a blended $33.6 \mathrm{~d} \mathrm{~EB}$ on an eccentric orbit $\left(\mathcal{H}_{E B, 33.6}^{e}\right)$. In all cases a grazing transit configuration is excluded.

A Dynamical Analysis The spectroscopic and blend analyses thus far leave us with three hypotheses. Here we will evaluate these hypotheses in light of the dynamical constraints from both the transit timing variations (TTV) and stability arguments.

It has been established that the observed large transit timing variations cannot be caused by a moon. It is shown in the main text that the only plausible source for such large TTVs is a third body in the system orbiting close to the 5:3 orbital resonance. These fits do not allow us to directly measure the mass for the transiting body but do allow us to constrain the eccentricity to a high degree of confidence. Thus, the first hypothesis we may consider is the eccentric $33.6 \mathrm{~d}$ blended EB scenario, $\mathcal{H}_{E B, 33.6}^{e}$.

Eccentricity Constraints. The spectroscopic analysis can be used to provide a mass and radius for the star using stellar evolution models, as was done earlier. However, if a substantial amount of blended light is present then the inferred properties would be unreliable. This is particularly salient in light of the faintness of the target and the subsequent lower-than-normal SNR spectra obtained. However, even in the case of a substantial amount of blended light, we are confident that the star is a dwarf on or near the main-sequence. We consider a wide range of corresponding plausible stellar masses to be $0.1 M_{\odot} \leq M_{*} \leq 10.0 M_{\odot}$.

Using this mass range, we re-fit the TTVs each time varying all of the system parameters. After finding the maximum likelihood solution, we perturb the parameters in order to derive an upper limit on the orbital eccentricity of the transiting planet. Across the full stellar range, we find $e_{P}<0.02$ to $99.9 \%$ confidence. Note that the derived TTVs are insensitive to any blended 
light and thus this limit is robust for $B>1$.

We conclude that the hypothesis of an eccentric $33.6 \mathrm{~d}$ blended EB is highly improbable. This now leaves only two remaining hypotheses to explain the data: $\mathcal{H}_{P}$ and $\mathcal{H}_{E B, 33.6}^{c}$.

Mass Constraints. The two surviving hypotheses are identical in that they both have a body on a $33.6 \mathrm{~d}$ circular orbit eclipsing a main-sequence dwarf star on a non-grazing transit. The only difference is the amount of blended light $(B=1$ versus $B>1)$. In both cases, the eclipsing object is small ( $p<0.3$ to $99.99 \%$ confidence) and cool $\left(\mathcal{F}_{P} / \mathcal{F}_{*}<0.0089\right.$ to $3-\sigma$ confidence, which is robust for any $B$ value). The only plausible blend scenarios which could reproduce all of these constraints is a very cool M-dwarf, a brown dwarf or a larger planet. Clearly the planetary nature of the transiting body is not yet validated as these objects span a wide range of possible masses.

The final and most powerful tool at our disposal is that the TTVs allow us to measure $M_{c} / M_{*}$ for the third body. For a given $M_{*}$ then, two of the three masses in the system are known, along with their periods, semi-major axes, eccentricities and mutual inclination. The only unknown is the mass of the transiting object. We may place an upper limit on this value by iteratively increasing the mass until the system becomes dynamically unstable.

Dynamical stability was investigated using the symplectic N-body code known as SyMBA (20), simulating the system for $1 \mathrm{Gyr}$ using an integration timestep of $1.5 \mathrm{~d}$. One can account for the possibility that the stellar mass derived from the spectroscopy is unreliable (due to blending) by investigating a wider, but plausible, range of stellar masses. To this end, we scanned the range $0.8 M_{\odot} \leq M_{*} \leq 1.2 M_{\odot}$. In order to be stable for $1 \mathrm{Gyr}$, we estimate $M_{P} / M_{*}<5 M_{J},<7 M_{J}$ and $<9 M_{J}$ for $0.8 M_{\odot}, 1.0 M_{\odot}$ and $1.2 M_{\odot}$ respectively, all of which exhibit a very sharp stability boundary. For our best-fit stellar mass of $0.9 M_{\odot}$ (see earlier spectroscopy discussion), the mass of KOI-872.01 is constrained to be $M_{P}<6 M_{J}$.

We therefore conclude that the transiting object KOI-872.01 must be planetary in nature (since $M_{P}<11 M_{J} ;(53)$ ) and thus refer to the object as KOI-872b from here-on-in. KOI- 
$872 \mathrm{c}$ is validated as a planet based upon the precise measurement of its mass from the TTVs, specifically $M_{c} / M_{*}=3.97_{-0.14}^{+0.17} \times 10^{-4}$, corresponding to $M_{c}=0.376_{-0.020}^{+0.023} M_{J}$.

A Search for Additional Transits Our analysis of the transit timing variations (TTVs) leads us to conclude that a second planet exists in the system with an orbital period of $57 \mathrm{~d}$. Given that the inner planet transits, and conclusion of nearly coplanar orbits from the TTV fits, there seems a reasonable hope for detecting transits of a second planet. However, given the fact KOI$872 \mathrm{c}$ is Saturn-mass, it should be a gas giant with a sizeable transit, roughly of the same size as KOI-872b. Such a transit would be easily spotted even by eye but there is no evidence for such events. Fig. S6 shows the phased data, accounting for the TTVs of KOI-872c upon the time of expected transit. Since the TTVs of KOI-872c depend upon the unknown mass of KOI-872b, we present 11 different realizations for various masses of planet $b$. In all cases no transit-like event is detectable.

Despite the fact KOI-872c does not seem to transit, we initiated a search for additional transits in the system. The light curve was searched for transits using the Box Least-Squares method (54). After removing the transits of KOI-872b, we detected a significant signal (SNR 14 ) in the light curve with an apparent depth of $\sim 0.3 \mathrm{mmag}$, and a period of $P=6.7668293 \mathrm{~d}$. The drop in brightness had a first-to-last-contact duration, relative to the total period, of $q=$ 0.0219 , corresponding to a total duration of $3.6 \mathrm{hr}$. The transit candidate is hereby referred to as KOI-872.03.

Transit Fit of KOI-872.03 Initial inspection of depth, duration and period for the KOI-872.03 transits suggested a physically plausible signal. Given the high significance of the signal, we investigated further by performing a full transit light curve fit accounting for the limb darkening of the star, the variable diluted light factors and the finite integration time of the long-cadence data, all of which are ignored in the BLS search.

We trim the data set to be within $\pm 0.5 \mathrm{~d}$ of the expected transit times, as computed from 
the BLS peak, in order to reduce the computation time. The 74 transit epochs require 74 OOT parameters to fit in conjunction with the transit parameters themselves. This large number of parameters makes a MultiNest fit unfeasible. Instead, we use a Markov Chain Monte Carlo (MCMC) routine with the Metropolis-Hastings rule. We executed two independent fits, one using a prior on $\rho_{*}$ from the $\mathcal{M}_{T}$ fit and one with a uniform prior on $\rho_{*}$ between the boundaries for a main-sequence star.

With the free $\rho_{*}$ fit, we detect a transit corresponding to a planet of size $\left(R_{P} / R_{*}\right)^{2}=$ $278_{-32}^{+26} \mathrm{ppm}(8.8 \sigma)$. With the prior $\rho_{*}$ this becomes $\left(R_{P} / R_{*}\right)^{2}=274_{-28}^{+26} \mathrm{ppm}(9.9 \sigma)$, corresponding to a planet of size $R_{P}=1.70_{-0.11}^{+0.11} R_{\oplus}$. The maximum likelihood realization of this latter fit is shown in Fig. S7 and the corresponding parameters estimates are provided in Table 1.

As expected, the prior- $\rho_{*}$ fit retrieves virtually the same $\rho_{*}$ as derived from planet $\mathbf{b}$, specifically $\rho_{*}=1560_{-150}^{+150} \mathrm{~kg} \mathrm{~m}^{-3}$. The impact parameter converges to $b=0.39_{-0.12}^{+0.19}$ corresponding to $i=\left(88.55_{-0.49}^{+0.69}\right)^{\circ}$. Curiously though, releasing this prior yields $\rho_{*}=1820_{-490}^{+660} \mathrm{~kg} \mathrm{~m}^{-3}$, $b=0.02_{-0.44}^{+0.44}$ and $i=\left(88.92_{-1.26}^{+0.75}\right)^{\circ}$. In other words, without any prior information, the light curve yields a consistent stellar density (within $0.6 \sigma$ ), highly indicative that KOI-872.03 orbits the same star as KOI-872b with negligible eccentricity.

Due to the low signal-to-noise, we were not able to determine individual transit times or durations for this object. We note that the expected TTVs of KOI-872b due to KOI-872.03 is less than 1 second in amplitude and thus undetectable with the current data. Further, the expected TTVs of KOI-872.03 are around $10 \mathrm{~s}$, which are again too small to detect. Without TTVs, we cannot causally link the object to be transiting the same host star as KOI-872.03 (it could be transiting a background star). Even assuming it was in the same system stability limits allow the object to be as massive as $100 M_{J}$ and still be stable. Although formally unconfirmable, the derived $\rho_{*}$ suggests it is likely associated with the same star. 
A Search for an Exomoon As discussed earlier, the observed TTVs cannot be adequately explained by an exomoon and only a second planet in the 5:3 resonance offers a valid solution. The presence of this second planet complicates our search for an exomoon. Further, the presence of stellar activity makes spot crossings and transit distortions probable, further exacerbating our search for a moon. Despite this, we here describe our efforts to search for an extrasolar moon.

Fitting for a Moon Eclipse After Removing the TTVs, $\mathcal{M}_{M T 1, M 0}$. The first attempt we made was to forcibly remove the best-fit TTVs from the time series and then fit for a zero-mass moon. This fit allows for a finite radius moon and thus is merely a search for the moon eclipse. We perform two versions of the model fit; one setting the exomoon radius to zero and one allowing the parameters $R_{S} / R_{P},\left(\rho_{P}\right)^{2 / 3}, P_{S}, \phi_{S}, i_{S}$ and $\Omega_{S}$ to be freely varied (see (38) for various definitions of these terms). The two models are performed so that we have a null model to compare against.

The results yield $\log \mathcal{Z}=(12001.34 \pm 0.37)$ for the null fit and $\log \mathcal{Z}=(12025.89 \pm 0.24)$ for the moon-transit fit, or a 6.7- $\sigma$ preference for the moon-transit model. Whilst certainly above our statistical significance threshold, one should recall that the star is active and these fitted events could merely be star spot crossings or activity-related events. Such events would be poorly sampled with the 30 minute cadence of the current observations though.

The mass-ratio of the planet and the star may be determined using the light curve alone, as described in (55). For our estimate of the stellar mass, this allows us to compute $M_{P}$ directly. The results find that $M_{P}>20 M_{J}$ for all modes, which exceeds the $6 M_{J}$ stability limit imposed on the system. We therefore conclude that none of these modes are genuine and most likely due to the presence of stellar activity. The likely presence of these spots also prevent us placing an upper excluded limit on a putative exomoon radius.

Fitting for a Moon After Removing Planet TTVs, $\mathcal{M}_{M T 2, R 0}$. We also tried removing the maximum likelihood TTVs from the planet-fit of KOI-872c. This is therefore a fit on the residual TTVs for a moon signal. Since the eclipse signal of the moon is likely unreliable 
due to stellar activity, we limit this search to a model, where the moon is a point-mass (i.e. $R_{S}=0$ ); model $\mathcal{M}_{M T 2, R 0}$. We found this model unable to locate a significantly improved fit with $\Delta \log \mathcal{Z}=-(1.55 \pm 0.44)$, relative to the null hypothesis. To illustrate this, Fig. [S8] shows the TTV residuals after removing the TTVs of KOI-872c along with the maximum likelihood model TTVs from $\mathcal{M}_{M T 2, R 0}$. Although no exomoon is detected, we can use the results to place upper limits on a putative exomoon mass. This is particularly valuable given that radius limits are not possible due to the likely presence of spots. Fig. S9 provides the corresponding mass limits, excluding $M_{S} / M_{P}<0.021$ to $3-\sigma$ confidence.

TTV Constraints on Additional Planets When the computed TTVs corresponding to s1 are subtracted from the measured TTVs, this leaves a small residual signal with a $\simeq 1$ minute amplitude. This is comparable to the measurement errors.

The small amplitude of the TTV residuals can be used to place limits on the presence of additional planets in the system. Given that KOI-872b and KOI-872c have nearly coplanar orbits, we tested a case in which the additional planet was placed in the invariant plane of the two confirmed planets. The orbits were followed by an $N$-body integrator to see whether the computed TTVs are consistent with the residuals.

The results are illustrated in Figs. S11 and S12, The small amplitude of residual TTVs provides an useful constraint on the third planet's mass and orbit. They rule out, for example, a Jupiter-mass planet on low- $e$ orbit with $0.05<a<0.5 \mathrm{AU}$.

Additional constraints can be obtained from the stability requirements. For example, a lowmass planet with $e \simeq 0$ and $i \simeq 0$ should have $\left|a-a_{c}\right| / a_{c}>C\left(M_{c} / M_{*}\right)^{2 / 7}$, where $M_{c}$ and $a_{c}$ are the mass and semimajor axis of KOI-872c, for the system to be stable (56), where $C \simeq 1.5$ (e.g., (57, 58)). For $M_{c} / M_{*}=4 \times 10^{-4}$, this gives $\left|a-a_{c}\right| / a_{c}>0.160$.

A more accurate stability criterion was derived in (59). For $M_{c} / M_{*}=4 \times 10^{-4}$ and $e_{c}=$ 0.015 , this criterion gives $\left|a-a_{c}\right| / a_{c}>1.8 e_{c}^{1 / 5}\left(M_{c} / M_{*}\right)^{1 / 5}=0.162$. The difference between 
the two criteria is therefore negligible in our case. As the mean motion resonances become wider with planet's eccentricity, the eccentricity-dependent criterion of (59) should be used for larger $e$. 


\section{References and Notes}

1. J. A. Carter, J. C., Yee, J. Eastman, S. B. Gaudi, J. N. Winn, ApJ, 689, 499 (2008)

2. J. Miralda-Escudé, ApJ, 564, 1019 (2002).

3. E. Agol, J. Steffen, R. Sari, W. Clarkson, MNRAS 359, 567 (2005).

4. M. J. Holman, N. W. Murray, Science, 307, 1288 (2005).

5. P. Sartoretti, J. Schneider, $A \& A S, \mathbf{1 3 4}, 553$ (1999).

6. D. M. Kipping, MNRAS, 392, 181 (2009a).

7. D. M. Kipping, MNRAS, 396, 1797 (2009b).

8. D. M. Kipping et al., ApJ, 750, 115 (2012).

9. W. J. Borucki et al., ApJ, 736, 19 (2011).

10. Materials and methods are available as supplementary material on Science Online.

11. F. Feroz \& M. P. Hobson, MNRAS 384, 449 (2008).

12. F. Feroz, M. P. Hobson, M. Bridges, MNRAS 398, 1601 (2009).

13. M. Montalto, $A \& A, \mathbf{5 2 1}, 60$ (2010).

14. C. A. Scharf, ApJ, 661, 1218 (2007).

15. C. A. Watson \& T. R. Marsh, MNRAS, 405, 2037 (2010).

16. R. R. Rafikov, ApJ, 700, 965 (2009).

17. D. Nesvorný, C. Beaugé, ApJL , 709, L44 (2010). 
18. D. Nesvorný, A. Morbidelli, ApJ, 688, 636 (2008).

19. We also tested the possibility of planets in the 1:1 mean motion resonance. We used an $N$ body integrator in this case, because perturbation theory described in (18) is not convergent for crossing orbits.

20. M. J. Duncan, H. F. Levison, M. H. Lee, AJ, 116, 2067 (1998).

21. To demonstrate this we generated $10^{6}$ realizations in the neighborhood of $\mathrm{s} 2$ and selected those consistent with the observed TTVs (as defined by $\chi^{2}<\chi_{\min , \mathrm{s} 2}^{2}+\delta \chi^{2}(99 \%)$ ). We

found that the mass and orbit were well constrained from TTVs with $M_{c} \simeq 1.8 \times 10^{-3} M_{*}$, $81.5<P_{c}<82.3 \mathrm{~d}, 0.02<e_{c}<0.05$ and $7^{\circ}<I_{c}<12^{\circ}$. We then calculated TDVs for the selected realizations and found that all were inconsistent with the measurements by $>3 \sigma$. In contrast, the realizations near $\mathrm{s} 2$ that were consistent with the observed TDVs had $I_{c}<5^{\circ}$, and were inconsistent with the observed TTVs.

22. We attempted to fit the observed TTVs with a three-planet system. The obtained solutions recovered the two-planet case corresponding to $\mathrm{s} 1$ or s2, with the third planet being either too small or too distant to produce significant TTVs.

23. J. J. Lissauer et al., ApJS 197, 8 (2011a).

24. M. J. Holman et al., Science 330, 51 (2010).

25. J. J. Lissauer et al., Nature 470, 53 (2011b).

26. S. Ballard et al., ApJ, 743, 200 (2011b).

27. T. M. Brown et al., ApJ, 552, 699 (2001).

28. R. L. Gilliland et al., ApJL, 713, 160 (2010).

29. T. Mazeh, S. Faigler, $A \& A, \mathbf{5 2 1}, 59$ (2010). 
30. D. M. Kipping, G. A. Bakos, ApJ, 730, 50 (2011).

31. D. M. Kipping, G. A. Bakos, ApJ, 733, 36 (2011).

32. N. Ahmed, T. Natarajan, K. R. Rao, IEEE Trans. Computers, 23, 90 (1974).

33. D. M. Kipping, D. S. Spiegel, MNRAS, 417, L88 (2011).

34. D. M. Kipping, G. Tinetti, MNRAS, 407, 2589 (2010).

35. E. B. Ford et al., ApJS, 197, 2 (2011).

36. E. B. Ford et al., ApJ, preprint available at http://arxiv.org/abs/1201.1892.

37. J. Skilling, in Fischer R., Preuss R., Toussaint U. V., eds, American Institute of Physics Conference Series Nested Sampling, pp 395405 (2004).

38. D. M. Kipping, MNRAS, 416, 689 (2011).

39. R. Kurucz, Stellar Model and Associated Spectra (http://kurucz.harvard.edu/grids.html) (2006).

40. A. Claret, $A \& A, \mathbf{3 6 3}, 1081$ (2000).

41. J. Díaz-Cordovés, A. Claret, A. Giménez, $A \& A S, 110,329$ (1995).

42. E. Agol et al., ApJ, 721, 1861 (2010).

43. T. D. Morton, J. A. Johnson, ApJ, 738, 170 (2011).

44. N. Batalha et al., ApJ, 713, 103 (2010).

45. D. Mislis, S. Hodgkin, MNRAS, preprint available at http://arxiv.org/abs/1202.1760.

46. S. K. Yi, ApJS, 136, 417 (2001). 
47. R. L. Kurucz, Model Atmospheres for Population Synthesis in Barbuy B., Renzini A., eds, Proc. IAU Symp. 149 p. 225 (1992).

48. S. Seager, G. Mallén-Ornelas, ApJ, 585, 1038 (2003).

49. D. M. Kipping, MNRAS, 407, 301 (2010).

50. G. Torres et al., ApJ, 614, 979 (2004).

51. F. Fressin et al., ApJS, 197, 5 (2011).

52. J. A. Carter, J. N. Winn, R. Gilliland, M. J. Holman, ApJ, 696, 241 (2009).

53. D. S. Spiegel, A. Burrows, J. A. Milsom, ApJ, 727, 57 (2011).

54. G. Kovács, S. Zucker, T. Mazeh, A\&A, 391, 396

55. D. M. Kipping, MNRAS, 409, L119 (2010).

56. J. Wisdom, $A J, \mathbf{8 5}, 1122$ (1980).

57. A. C. Quillen, P. Faber, MNRAS, 373, 1245 (2006).

58. SE. Chiang, E. Kite, P. Kalas, J. R. Graham, M. Clampin, ApJ, 693, 734 (2009).

59. A. J. Mustill, M. C. Wyatt, MNRAS, 419, 3074 (2012). 


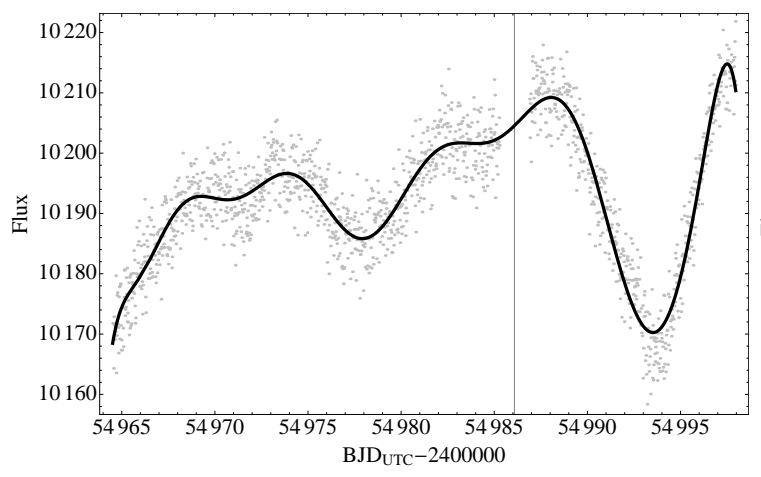

(a) Quarter 1

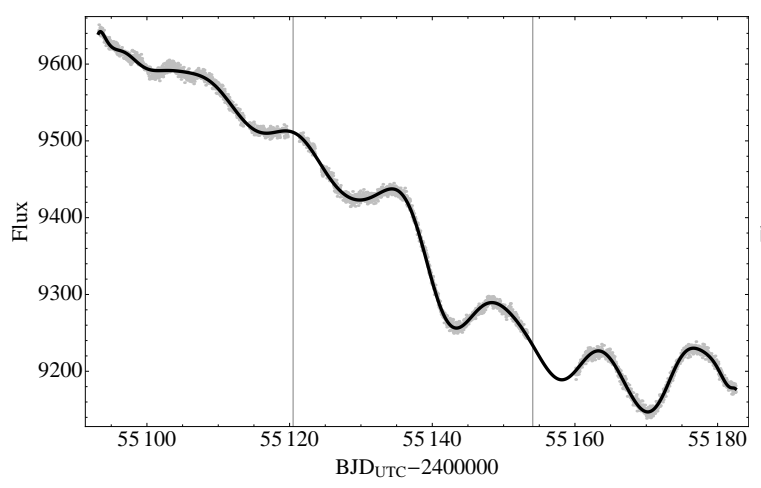

(c) Quarter 3

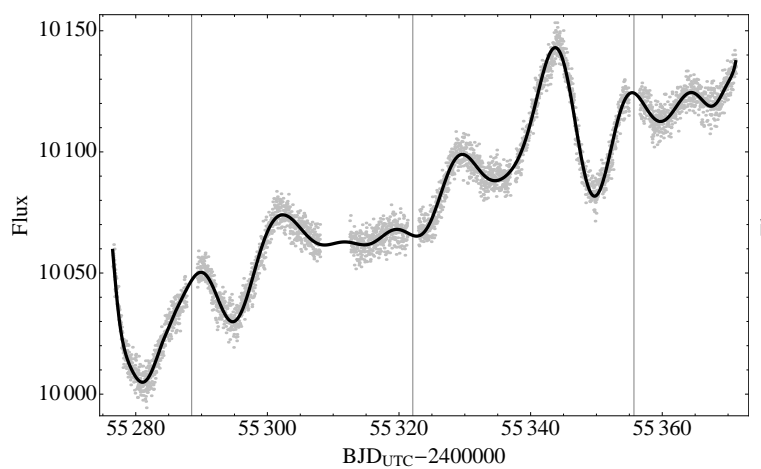

(e) Quarter 5

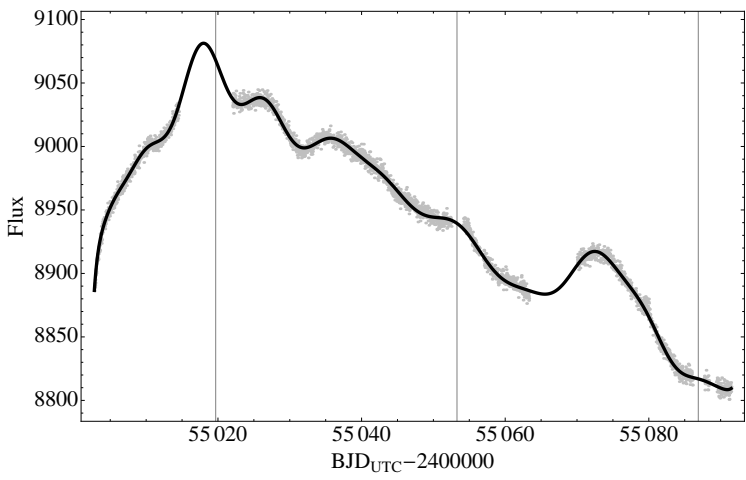

(b) Quarter 2

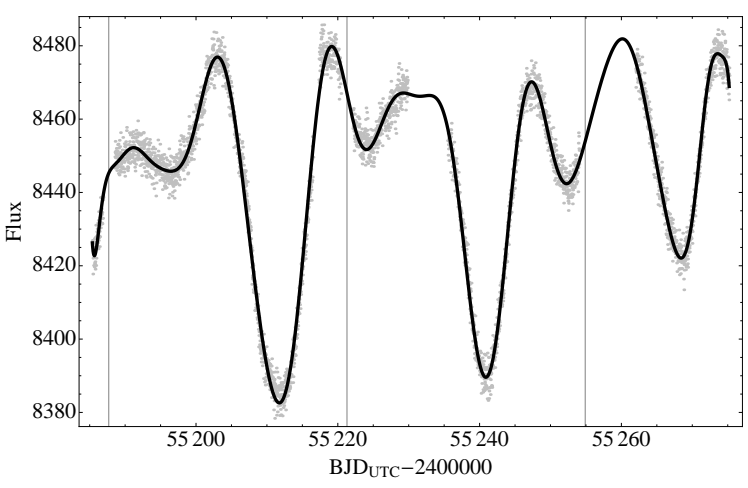

(d) Quarter 4

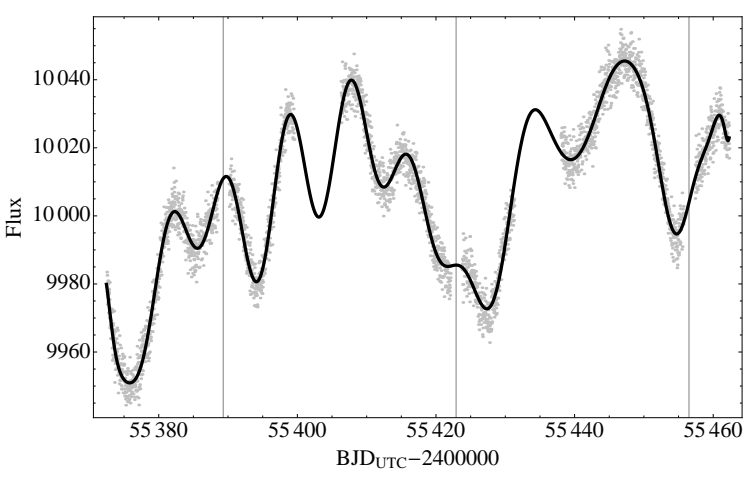

(f) Quarter 6

Figure S1: "Raw" (PA output) flux observed by Kepler from DR5 for Q1-6 of the source KOI-872 aka HCV/HCA-439. Overlaid is our model for the long-term trend, computed using a discrete cosine transform for each data set. Outliers and discontinuous systematic effects have been excluded. Transits (removed) marked with vertical gridlines. 


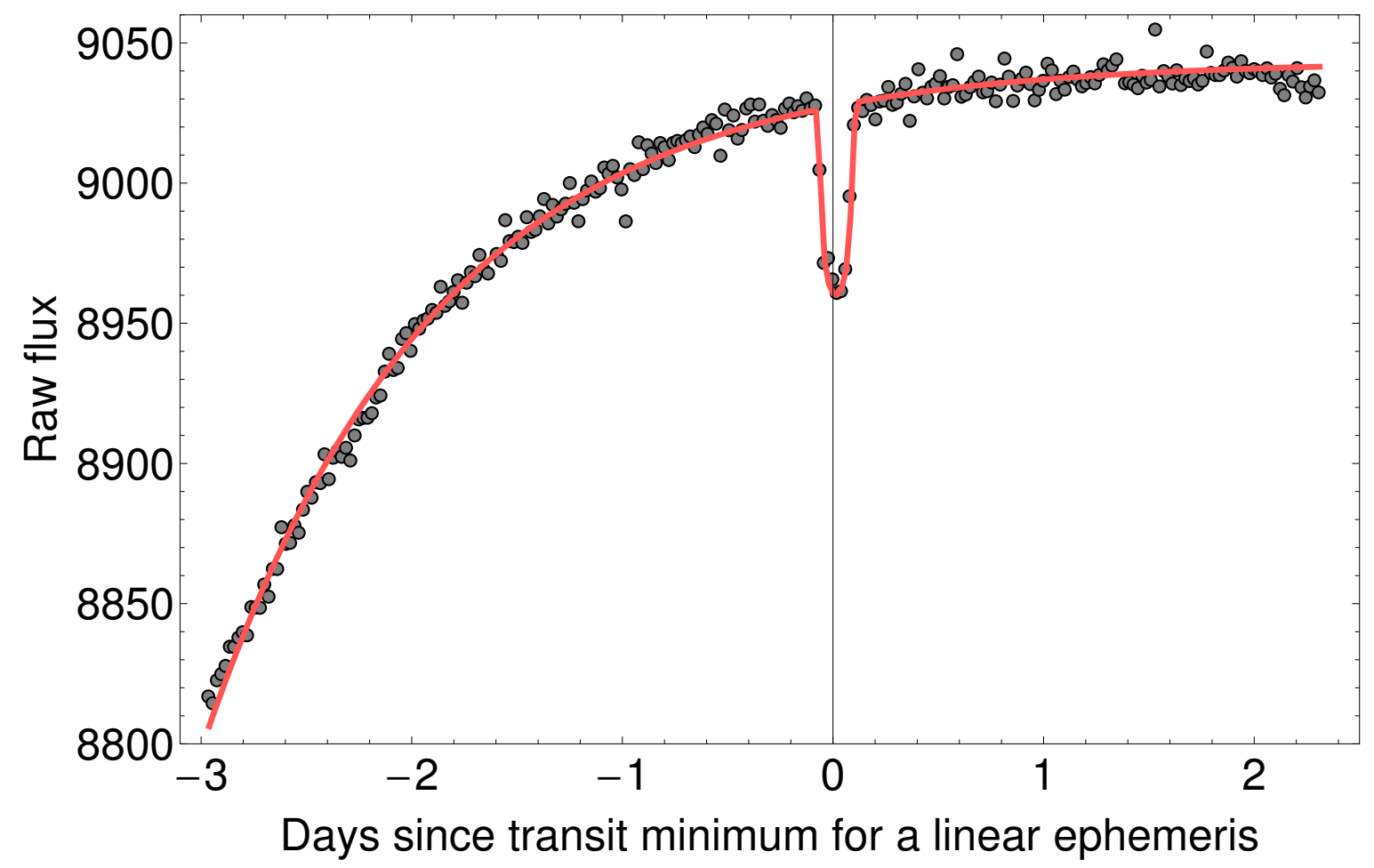

Figure S2: Maximum likelihood model (black line) overlaid with the long-cadence Kepler data for KOI-872, surrounding the ramp affected transit. The simple exponential ramp model is shown to provide an excellent description of the instrumental effect. 

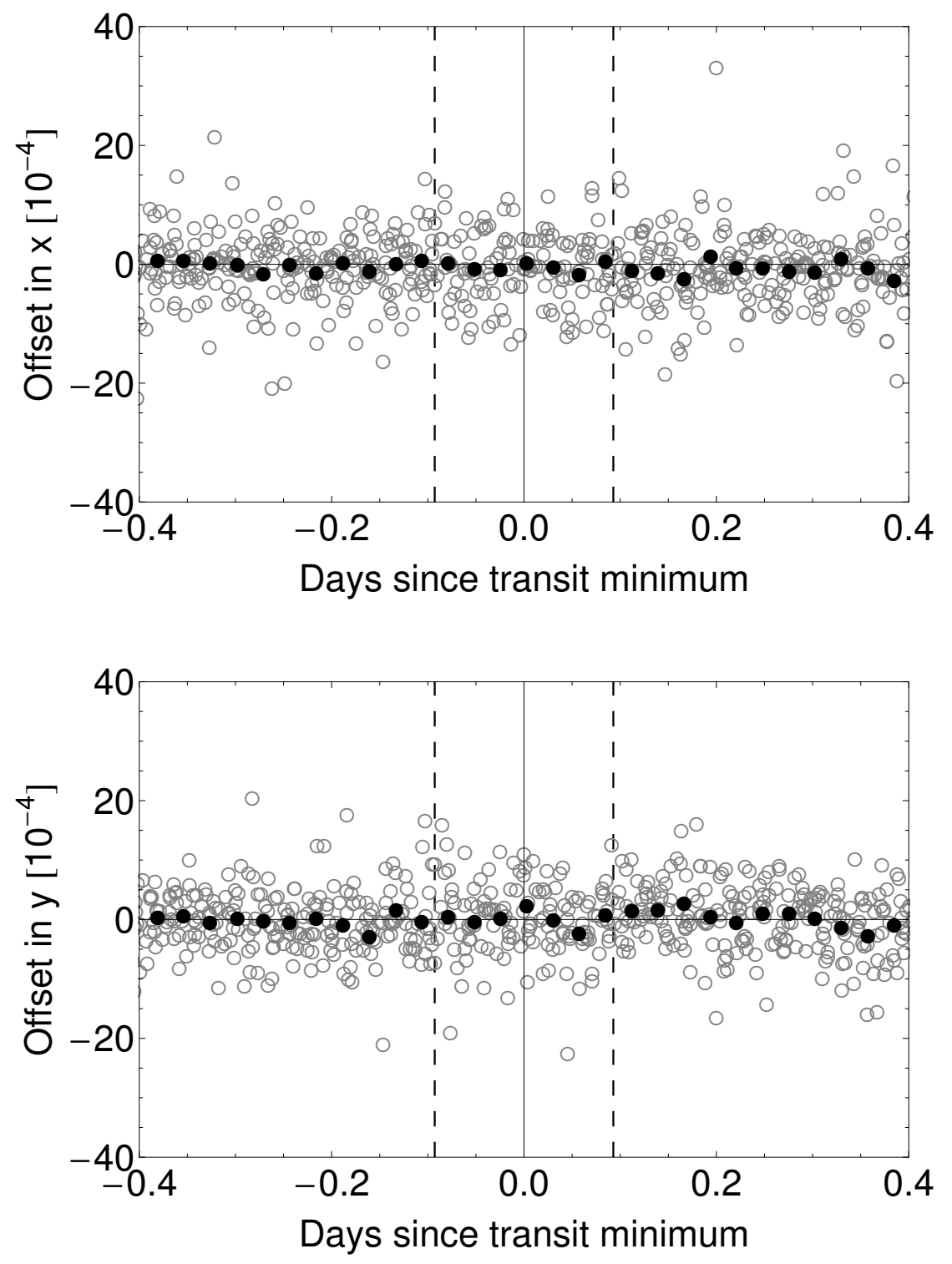

Figure S3: $x$ and $y$ centroid positions of KOI-872 relative to the median value over a 0.8 day range, surrounding the transits of KOI-872b. Data temporally offset and phased to account for the transit timing variations. The vertical grid lines mark the first and fourth contact points. We find no deviation of the centroids between in- versus out-of-transit, which would have indicated a separated blend source. 


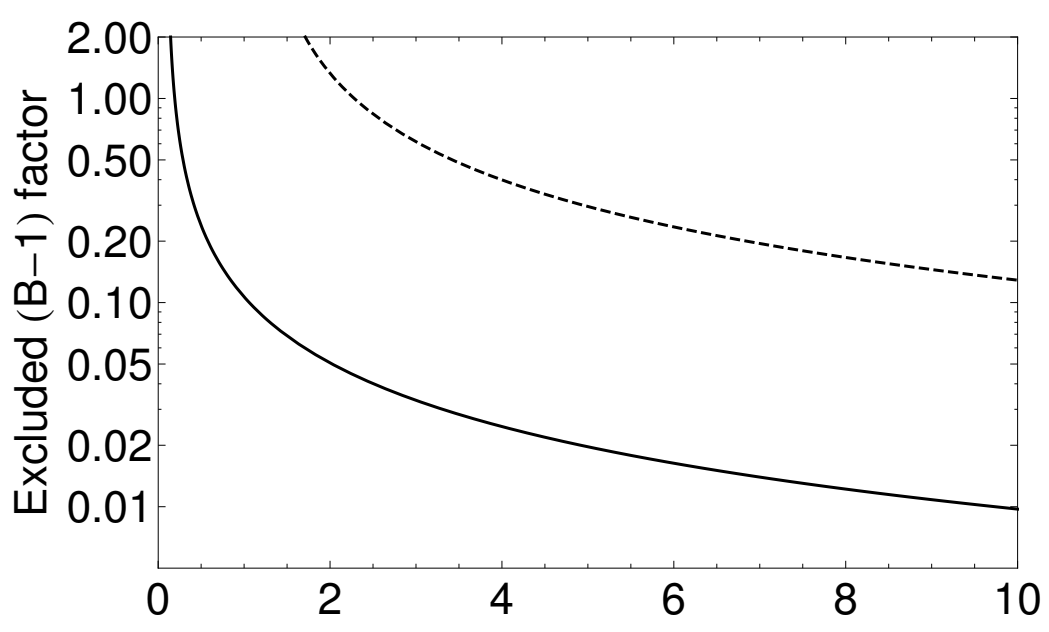

Sky-projected separation of companion ["]

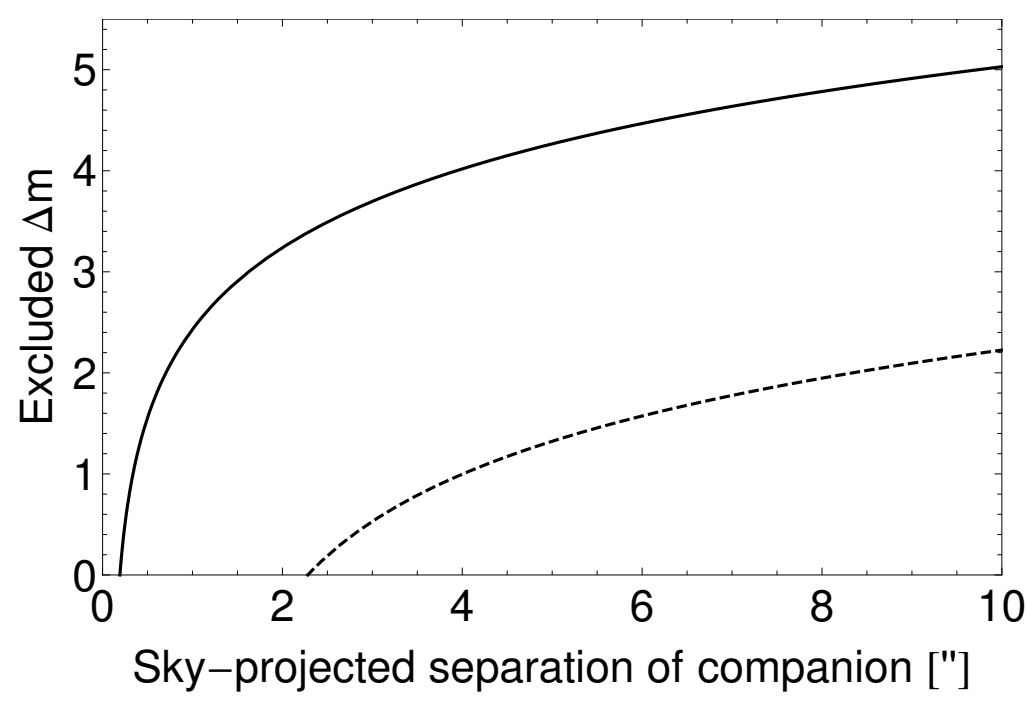

Figure S4: By comparing the in-transit to out-of-transit centroid position for KOI-872b (solid) and KOI-872.03 (dashed), we find no evidence for a shift corresponding to a separated blend source. Our upper limits allow us exclude the blend factor, $B$, as a function of the separation of a companion. The top panel shows the results when plotted for $B$, the lower panel when plotted for magnitude difference. 


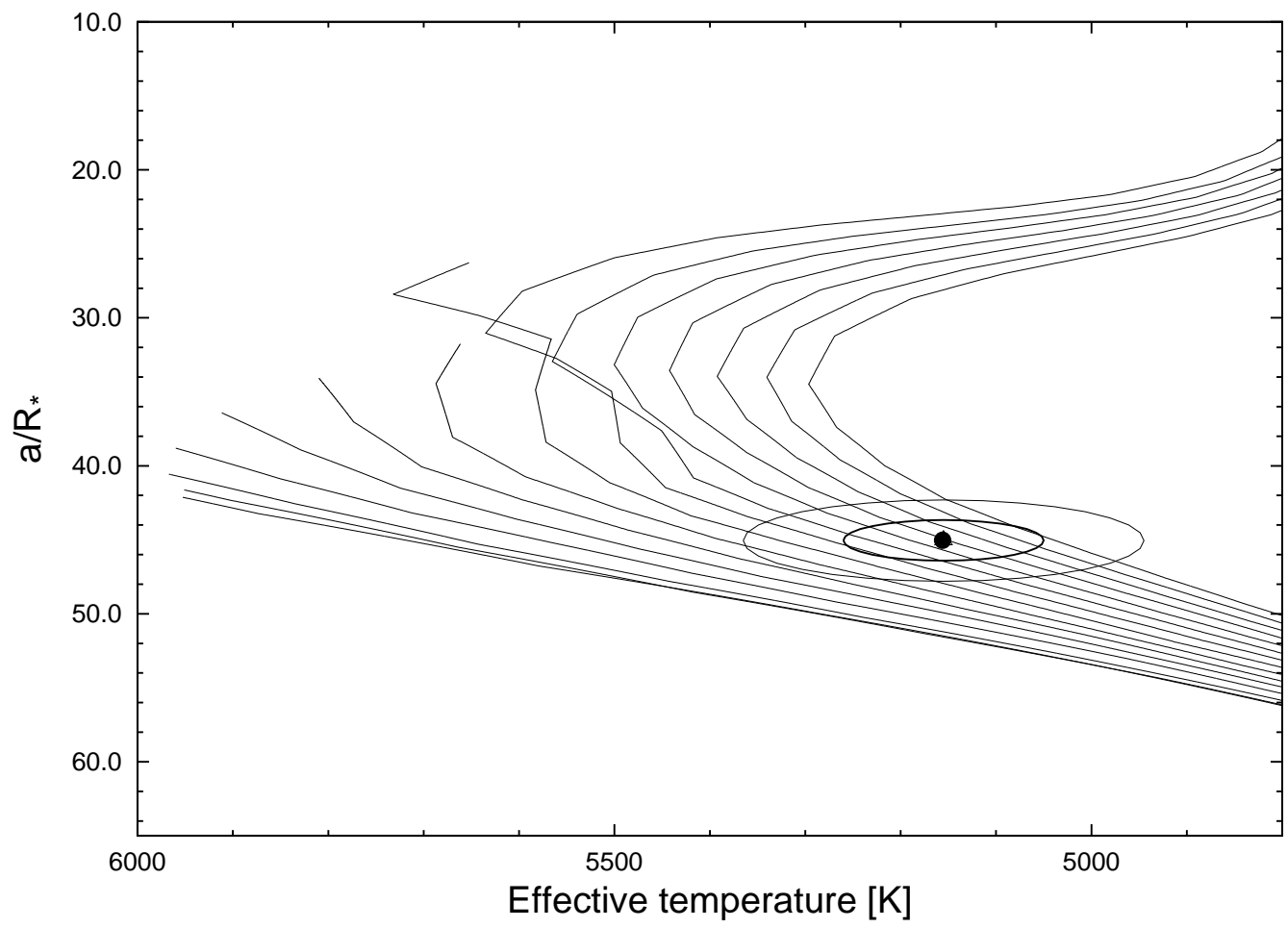

Figure S5: YY-isochrone analysis of KOI-872. Using our spectrosopic observations and the constraint on $\rho_{*}$ from the transit light curve of KOI-872b, we are able to determine precise parameters for the host star. The backdrop of isochrones is for ages $0.2,0.5,1.0,2.0, \ldots 13.0 \mathrm{Gyr}$ (from bottom to top) and $[\mathrm{Fe} / \mathrm{H}]=0.41$. The solution indicates an old star with $10 \pm 3 \mathrm{Gyr}$ age. 


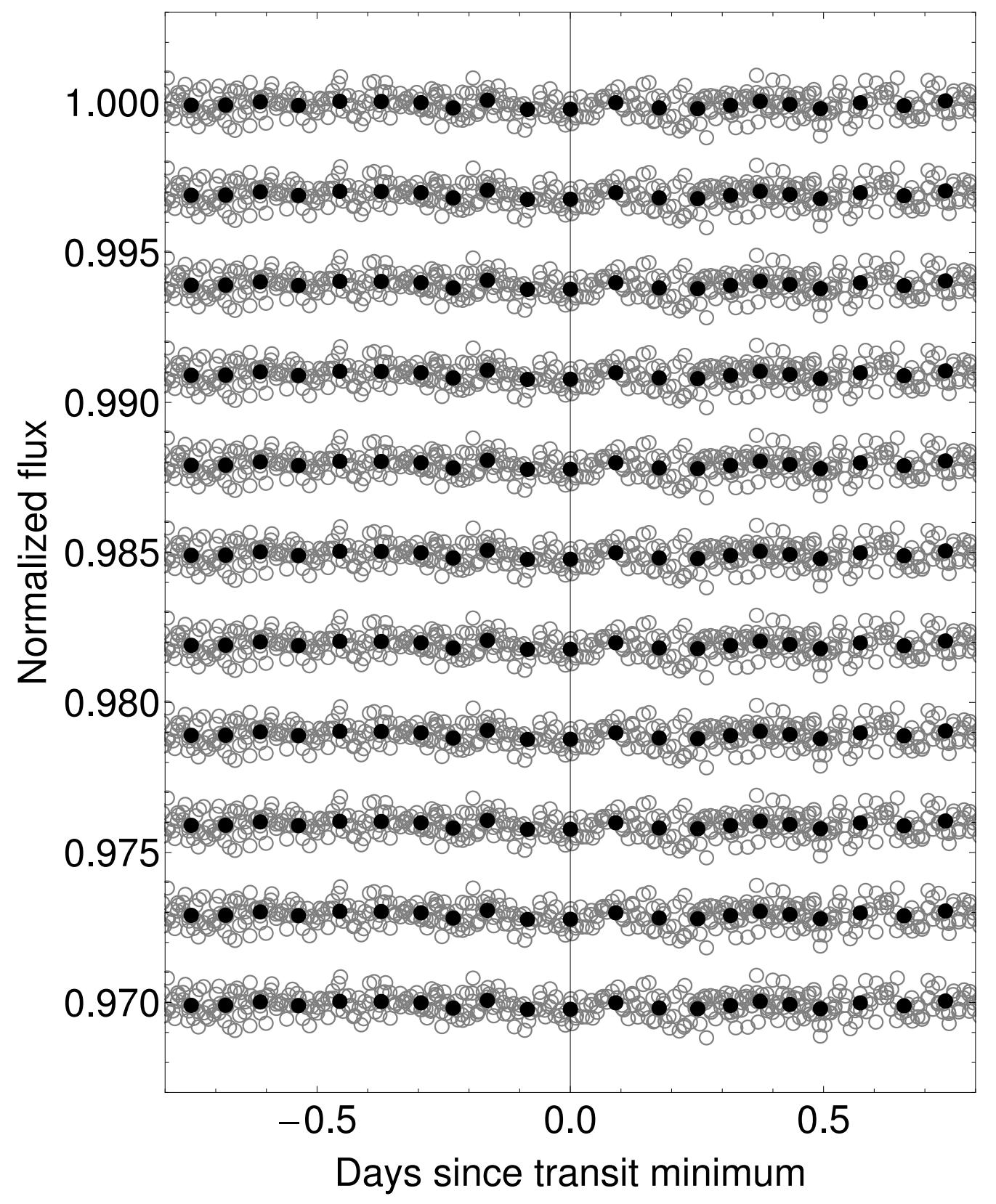

Figure S6: Using the TTVs of KOI-872b, we are able to predict the times of transit for KOI872c. Assuming masses for planet b ranging from 0 (top) to $6 M_{J}$ (bottom) for the primary in equal steps, we show the Kepler photometry phased upon these 11 candidate TTV ephemeres. Gray indicates the original data and black indicates 20-point binned data. We find no evidence for even a grazing geometry of KOI-872c. 


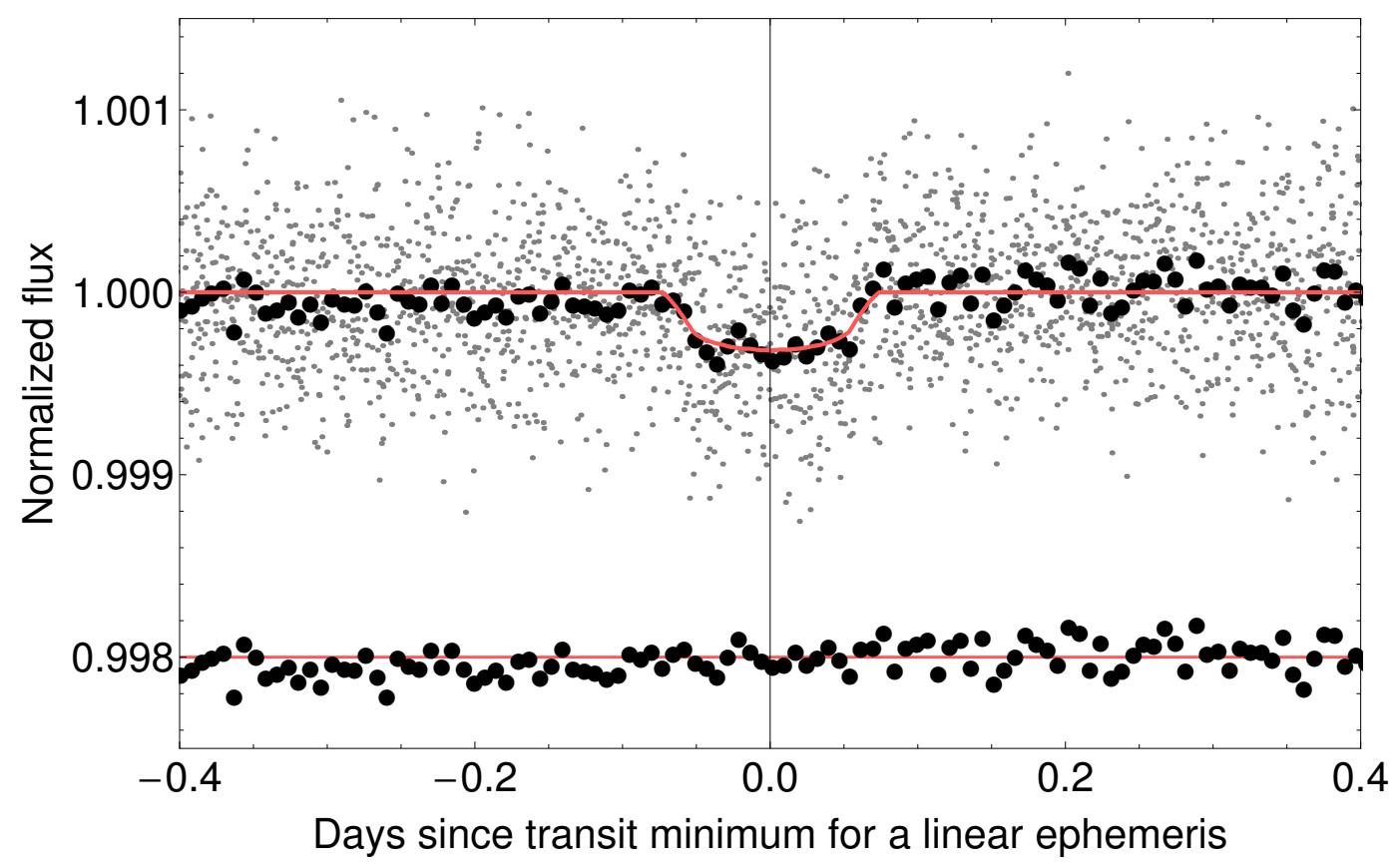

Figure S7: Maximum likelihood realization of a Markov Chain Monte Carlo fit for the transits of KOI-872.03. Gray points show original data and black is 20-point phase binned data. The maximum likelihood model is in red and the residuals are shown below offset at +0.998 . 


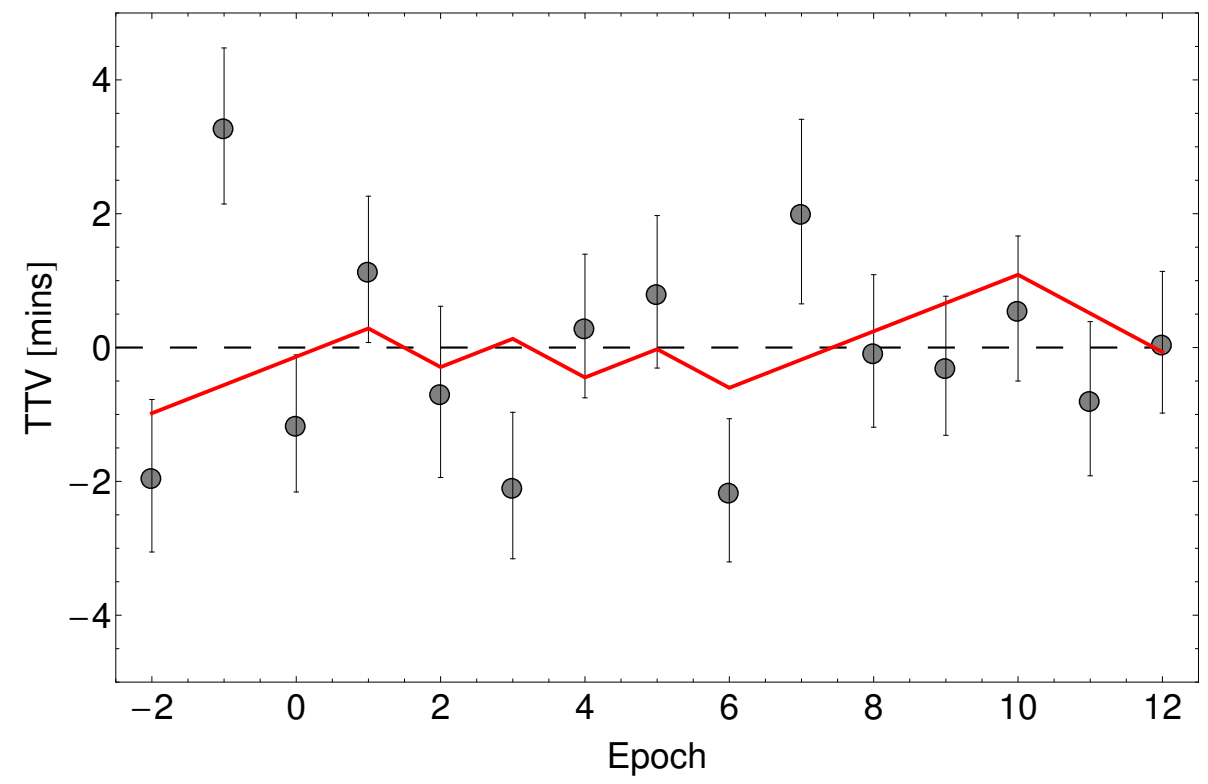

Figure S8: Maximum a-posteriori moon fit to the TTVs of KOI-872b (using model $\mathcal{M}_{M T 2, R 0}$ ), after removing the maximum likelihood TTVs due to the second planet, KOI-872c. We find no significant improvement by including a moon. 


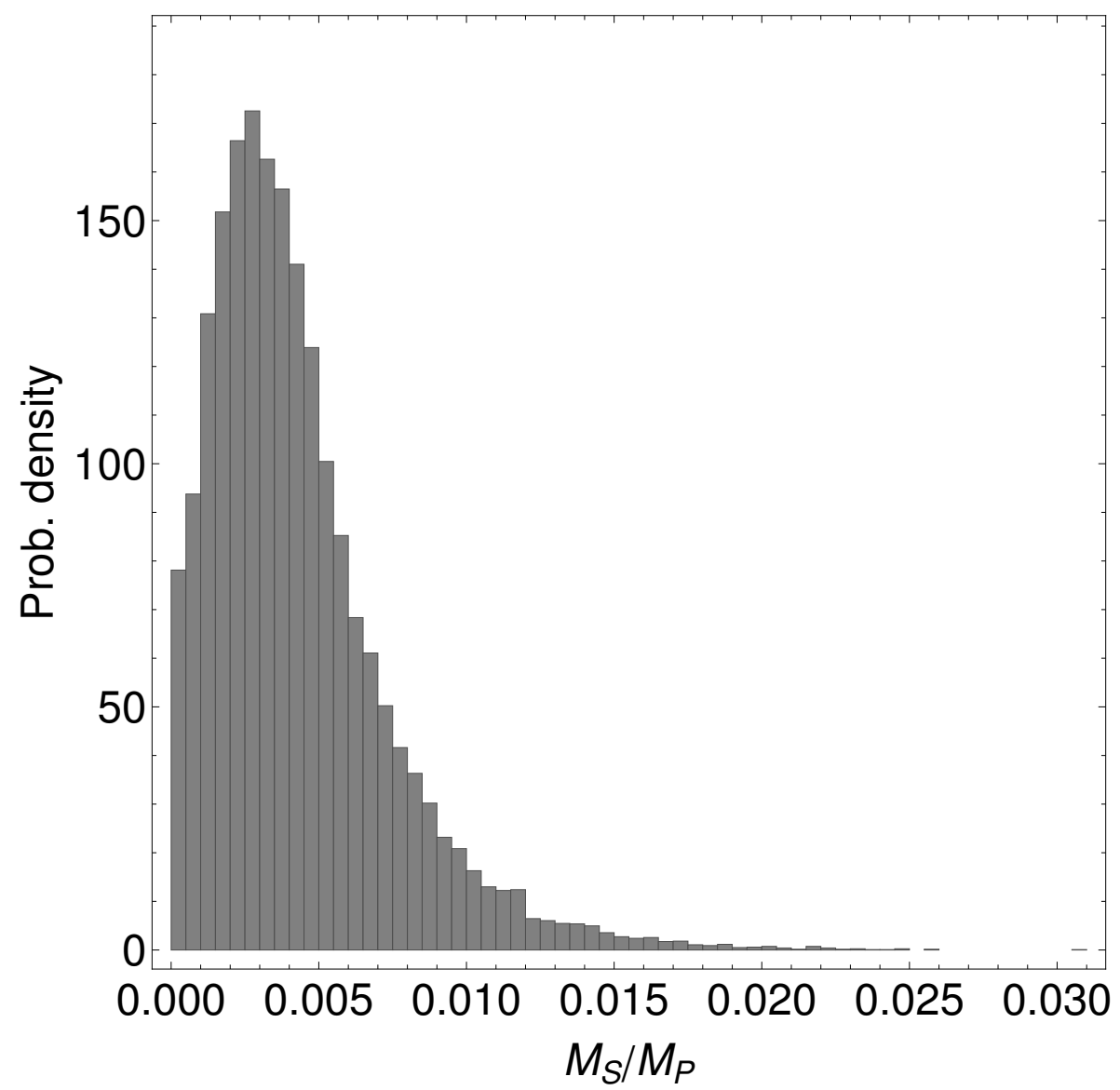

Figure S9: Posterior distribution for the mass of an exomoon relative to the mass of KOI-872b as computed using model $\mathcal{M}_{M T 2, R 0}$, marginalized over the entire prior volume. We estimate a 3- $\sigma$ upper limit of $M_{S} / M_{P}<0.021$ for this planet. 

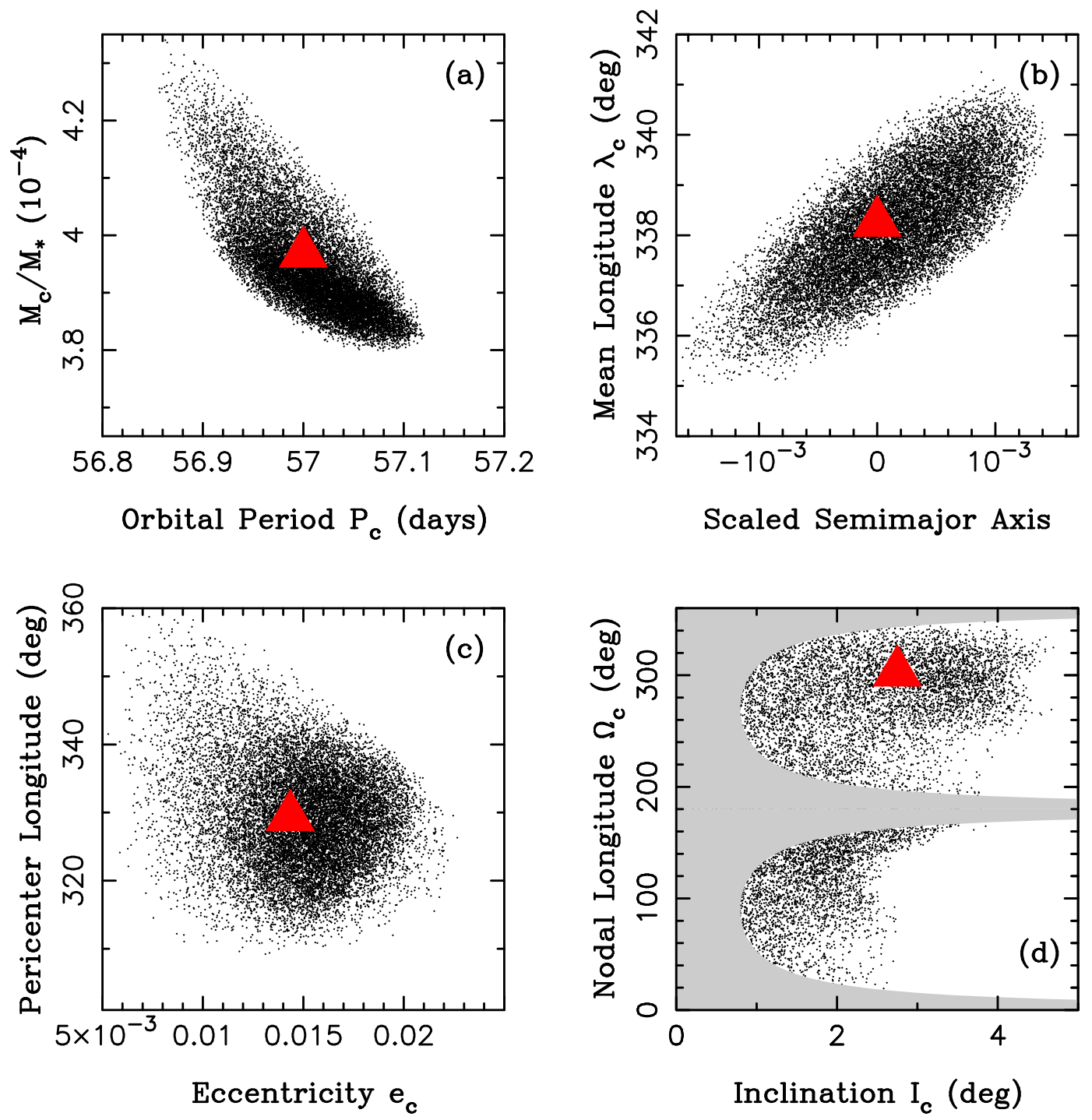

Figure S10: Confidence intervals. The dots denote the 99\% confidence area around s1 (red triangles). We sampled the general neighborhood of s1, determined $\chi^{2}$ for each parameter set (3 million parameter sets in total) and plotted a dot if $\chi^{2}<\chi_{\min }^{2}+\Delta \chi^{2}(99 \%)=23.5$. All parameters shown here are well constrained, including the orbital inclination of KOI-872c (the gray area in (d) is ruled out by the lack of KOI-872c's transits). This result was obtained while fixing $e_{b}=0$. A nearly identical result was obtained by letting $e_{b}$ (and $\varpi_{b}$ ) vary. In that case, $\chi^{2}<\chi_{\min }^{2}+\Delta \chi^{2}(99 \%)$ with $\Delta \chi^{2}(99 \%)=16.9$ for $15-9=6$ DOF gives $e_{b}<0.02$, leaving $\varpi_{b}$ unconstrained. The scaled semimajor axis in panel (b) is defined as $\left(a-a_{c}\right) / a_{c}$, where $a_{c}$ is the best-fit semimajor axis value for solution s1. 


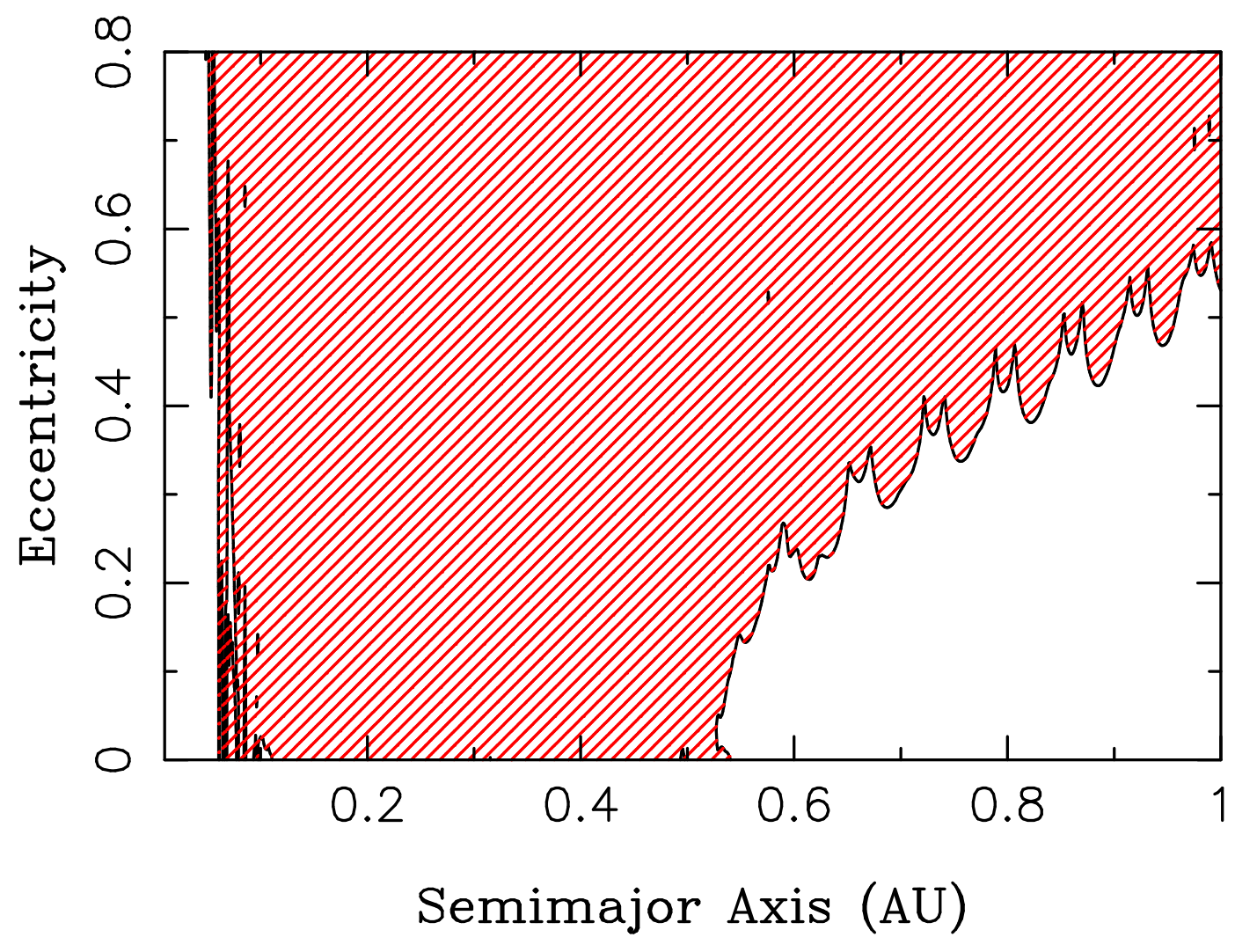

Figure S11: Constraints on the presence of an additional Jupiter-mass planet. The dashed area shows where the Jupiter-mass planet would induce the TTV amplitude of KOI-872b in excess of 1 minute. If the residual TTVs are caused by a Jupiter-mass planet, this planet should have $a$ and $e$ near the boundary of the shaded area. 


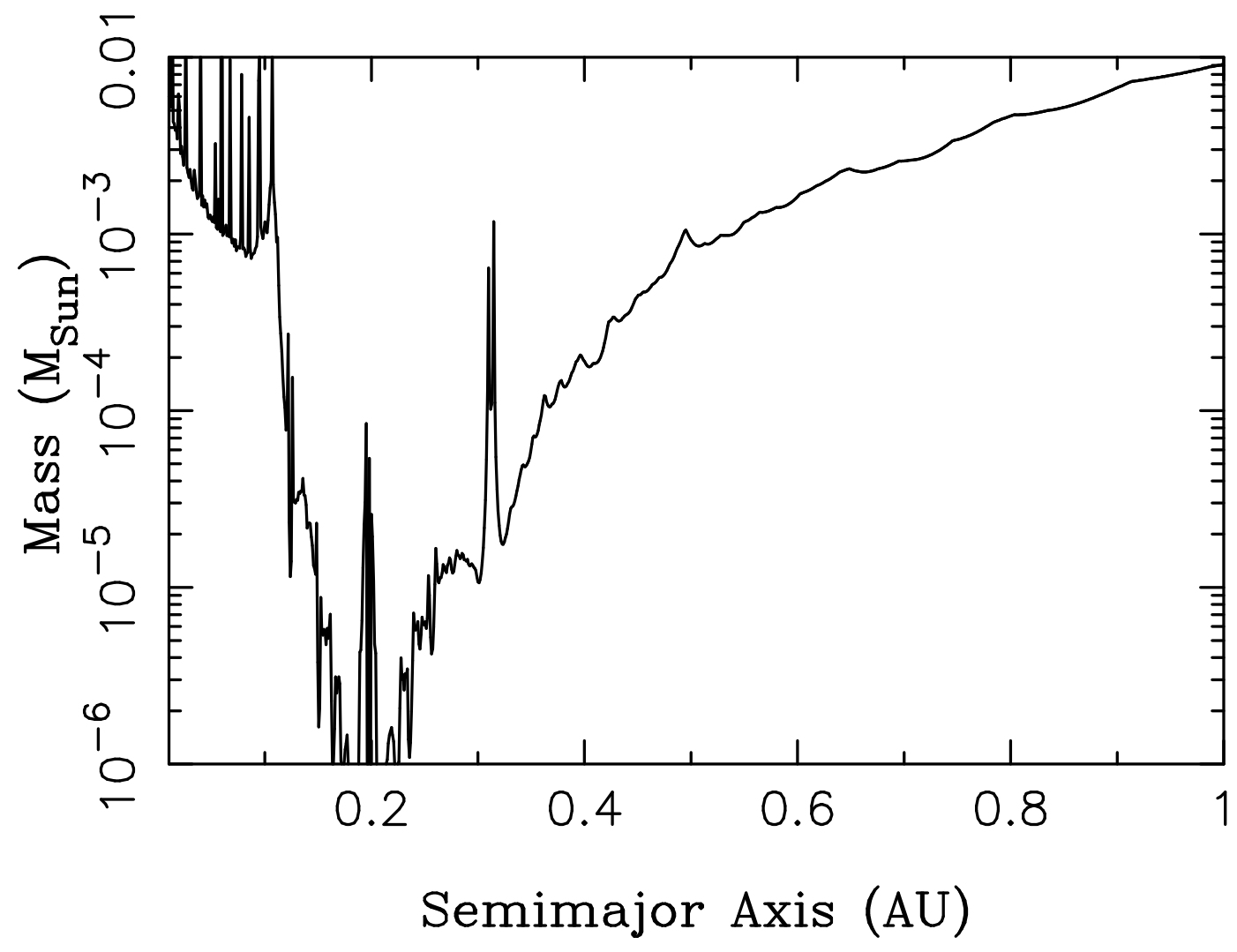

Figure S12: Constraints on the presence of an additional planet on circular orbit. The line shows where the planet induces the TTV amplitude of 1 minute. Note that the low-mass planet on a circular orbit exterior to KOI-872b cannot explain the TTV residuals, because such a planet would be perturbed by KOI-872c and would be dynamically unstable. 


\begin{tabular}{|c|c|c|c|}
\hline Parameter & $\mathcal{M}_{P}$ & $\overline{\mathcal{M}_{V} \& \mathcal{M}_{T}}$ & $\overline{\mathcal{M}_{M *}}$ \\
\hline$p^{\ddagger}$ & $\mathcal{U}[0,0.25]$ & $\mathcal{U}[0,0.25]$ & $\mathcal{U}[0,0.25]$ \\
\hline$\left[\rho_{*}^{\text {circ }}\right]^{2 / 3}\left[\mathrm{~kg}^{2 / 3} \mathrm{~m}^{-2}\right]^{\ddagger}$ & $\mathcal{U}[7.6499,6097.85]$ & $\mathcal{U}[7.6499,6097.85]$ & $\mathcal{U}[7.6499,6097.85]$ \\
\hline$b_{P}^{\ddagger}$ & $\mathcal{U}[0,1.25]$ & $\mathcal{U}[0,1.25]$ & $\mathcal{U}[0,1.25]$ \\
\hline$P_{P}[$ days $]$ & $\mathcal{U}\left[P_{P}^{*}-1, P_{P}^{*}+1\right]$ & $\mathcal{N}\left[P_{P}^{*}, 0.0002\right]$ & $\mathcal{U}\left[P_{P}^{*}-1, P_{P}^{*}+1\right]$ \\
\hline$\tau_{0}$ & $\mathcal{U}\left[\tau_{0}^{*}-1, \tau_{0}^{*}+1\right]$ & N/A & $\mathcal{U}\left[\tau_{0}^{*}-1, \tau_{0}^{*}+1\right]$ \\
\hline$a_{1}$ & $\mathcal{U}[150,250]$ & $\mathcal{U}[150,250]$ & $\mathcal{U}[150,250]$ \\
\hline$a_{2}$ & $\mathcal{U}[0.8,1.3]$ & $\mathcal{U}[0.8,1.3]$ & $\mathcal{U}[0.8,1.3]$ \\
\hline$O O T_{n}$ & $\mathcal{U}[0.95,1.05]$ & $\mathcal{U}[0.95,1.05]$ & $\mathcal{U}[0.95,1.05]$ \\
\hline$O O T_{-1}$ & $\mathcal{U}[8589.6487,9493.8223]$ & $\mathcal{U}[8589.6487,9493.8223]$ & $\mathcal{U}[8589.6487,9493.8223]$ \\
\hline$\tau_{n}$ & N/A & $\mathcal{U}\left[\tau_{n}^{*}-1, \tau_{n}^{*}+1\right]$ & N/A \\
\hline$e_{P}$ & $\delta[0]$ & $\delta[0]$ & $\delta[0]$ \\
\hline$\omega_{P}[\mathrm{rads}]$ & N/A & N/A & N/A \\
\hline$u_{1}$ & $\delta[0.3542]$ & $\delta[0.3542]$ & $\delta[0.3542]$ \\
\hline$\left(u_{1}+u_{2}\right)$ & $\delta[0.7140]$ & $\delta[0.7140]$ & $\delta[0.7140]$ \\
\hline$\sigma_{W}$ & $\mathcal{J}^{\prime}\left[\sigma_{\text {photo }}, 10 \sigma_{\text {photo }}\right]$ & $\mathcal{J}^{\prime}\left[\sigma_{\text {photo }}, 10 \sigma_{\text {photo }}\right]$ & $\mathcal{J}^{\prime}\left[\sigma_{\text {photo }}, 10 \sigma_{\text {photo }}\right]$ \\
\hline$\left(R_{S} / R_{P}\right)$ & N/A & N/A & $\mathcal{U}[0,1]$ \\
\hline$P_{S}[$ days $]$ & N/A & N/A & $\mathcal{U}[0.083,19.3944]$ \\
\hline$\phi_{S}[\mathrm{rads}]$ & N/A & N/A & $\mathcal{U}[0,2 \pi]$ \\
\hline$\left[\rho_{P}\right]^{2 / 3}\left[\mathrm{~kg}^{2 / 3} \mathrm{~m}^{-2}\right]$ & N/A & N/A & $\mathcal{U}[18.4982,920.9760]$ \\
\hline$i_{S}[\mathrm{rads}]$ & N/A & N/A & $\mathcal{U}[0,2 \pi]$ \\
\hline$\Omega_{S}[\mathrm{rads}]$ & N/A & N/A & $\mathcal{U}[-\pi / 2, \pi / 2]$ \\
\hline$\left[\rho_{S}\right]^{2 / 3}\left[\mathrm{~kg}^{2 / 3} \mathrm{~m}^{-2}\right]^{\dagger \times}$ & N/A & N/A & $\mathcal{U}[0,920.9760]$ \\
\hline$e_{S}$ & $\delta[0]$ & $\delta[0]$ & $\delta[0]$ \\
\hline$\omega_{S}[\mathrm{rads}]$ & N/A & N/A & N/A \\
\hline
\end{tabular}

Table S1: Priors used for various free parameters in the MultiNest fits. $\mathcal{U}[x, y]$ is a uniform prior between $x$ and $y . \mathcal{N}[x, y]$ is a Gaussian prior with mean $x$ and standard deviation $y . \delta[x]$ is a delta-function prior centered on $x . \mathcal{J}^{\prime}[x, y]$ is a modified Jeffrey's prior with an inflection point at $x$ and a maximum limit of $y$. We use the replacements $\tau_{0}^{*}=2455053.2815 \mathrm{BJD}_{\mathrm{UTC}}$, $P_{P}^{*}=33.6013 \mathrm{~d}$ and $\tau_{n}^{*}=\tau_{0}^{*}+n P_{P}^{*} . \sigma_{\text {photo }}$ is the median photometric error outputted from the Kepler pipeline for the time series under analysis. ${ }^{\ddagger}=$ for $\mathcal{M}_{V}$ fits these terms are independently fitted to each transit epoch. N/A = parameter is fixed to some arbitrary value, since it has no influence on the fits. ${ }^{\dagger}=$ for $\mathcal{M}_{M, R 0}$ and $\mathcal{M}_{M T 2, R 0}$, this term is replaced with $\left(M_{S} / M_{P}\right)$ and treated as uniform between 0 and $1 .{ }^{\times}=$for $\mathcal{M}_{M T 1, M 0}$, this term is fixed to zero to represent a zero-mass moon. 


\begin{tabular}{ll}
\hline \hline Model & $\log \mathcal{Z}$ \\
\hline Original data & \\
\hline $\mathcal{M}_{P}$ & $(10981.35 \pm 0.35)$ \\
$\mathcal{M}_{T}$ & $(117948.82 \pm 0.28)$ \\
$\mathcal{M}_{V}$ & $(11313.56 \pm 0.22)$ \\
$\mathcal{M}_{M}$ & $(11403.03 \pm 0.22)$ \\
$\mathcal{M}_{M, R 0}$ & \\
\hline All TTVs removed & $(12001.34 \pm 0.37)$ \\
\hline $\mathcal{M}_{M T 1, \text { null }}$ & $(12025.89 \pm 0.24)$ \\
$\mathcal{M}_{M T 1, M 0}$ & $(12006.43 \pm 0.37)$ \\
\hline Planet TTVs removed & $(12004.90 \pm 0.23)$ \\
\hline $\mathcal{M}_{M T 2, \text { null }}$ & \\
$\mathcal{M}_{M T 2, R 0}$ & \\
\hline \hline
\end{tabular}

Table S2: Bayesian evidences for the planetary system family of models fitted to the KOI872 photometry. The data favor model $\mathcal{M}_{T}$, a planet-only model with transit timing variations (TTV).

\begin{tabular}{|c|c|c|c|}
\hline Epoch & $\mathrm{OOT}_{i}$ & $\tau_{i}\left[\mathrm{BJD}_{\mathrm{UTC}}\right]$ & TTV [mins] \\
\hline-2 & $0.999732_{-0.000020}^{+0.00020}$ & $2454986.09325_{-0.00079}^{+0.00079}$ & $20.8_{-1.1}^{+.1}$ \\
\hline-1 & $9043.45_{-0.31}^{+0.30}$ & $2455019.69845_{-0.00081}^{+0.00081}$ & $26.4_{-1.2}^{+1.2}$ \\
\hline 0 & $1.000150_{-0.000020}^{+0.000020}$ & $2455053.29450_{-0.00071}^{+0.00073}$ & $18.7_{-1.0}^{+1.2}$ \\
\hline 1 & $0.995315_{-0.000020}^{+0.000020}$ & $2455086.86287_{-0.00076}^{+0.00075}$ & $-28.8_{-11}^{+1.1}$ \\
\hline 2 & $0.999953_{-0.000022}^{+0.000021}$ & $2455120.43870_{-0.00089}^{+0.00087}$ & $-65.5_{-1.3}^{+1.1}$ \\
\hline 3 & $1.004089_{-0.000041}^{+0.00040}$ & $2455154.07497_{-0.00076}^{+0.00076}$ & $-15.2_{-1.1}^{+1.1}$ \\
\hline 4 & $0.999651_{-0.000021}^{+0.000020}$ & $2455187.68561_{-0.00075}^{+0.00076}$ & $-1.9_{-1.1}^{+1.1}$ \\
\hline 5 & $0.999807_{-0.000021}^{+0.000020}$ & $2455221.33536_{-0.00079}^{+0.00081}$ & $67.8_{-1.1}^{+1.2}$ \\
\hline 6 & $1.000474_{-0.000020}^{+0.000021}$ & $2455254.90533_{-0.00074}^{+0.00078}$ & $22.7_{-1.1}^{+1.1}$ \\
\hline 7 & $0.999937_{-0.000020}^{+0.000020}$ & $2455288.46626_{-0.00096}^{+0.00096}$ & $-35.6_{-1.4}^{+1.4}$ \\
\hline 8 & $0.999864_{-0.000020}^{+0.00020}$ & $2455322.05975_{-0.00079}^{+0.00081}$ & $-46.9_{-1.1}^{+1.2}$ \\
\hline 9 & $0.999749_{-0.000020}^{+0.000020}$ & $2455355.66579_{-0.00072}^{+0.00072}$ & $-40.1_{-1.0}^{+1.0}$ \\
\hline 10 & $0.999834_{-0.000021}^{+0.000020}$ & $2455389.34350_{-0.00075}^{+0.00076}$ & $69.8_{-1.1}^{+1.1}$ \\
\hline 11 & $0.999762_{-0.000020}^{+0.000020}$ & $2455422.92851_{-0.00080}^{+0.00079}$ & $46.3_{-1.2}^{+1.1}$ \\
\hline 12 & $1.000111_{-0.000020}^{+0.00020}$ & $2455456.48567_{-0.00074}^{+0.00076}$ & $-17.3_{-1.1}^{+1.1}$ \\
\hline
\end{tabular}

Table S3: Epoch-specific fitted parameters for the KOI-872 system. Results computed from the weighted posteriors resulting from model $\mathcal{M}_{T}$, using MultiNest. TTVs relative to maximum a-posteriori linear ephemeris derived in model fit $\mathcal{M}_{P} ; P_{P}=33.6013506 \mathrm{~d}$ and $\tau_{0}=2455053.2815010$. Physical system parameters are provided in Table 1 . 


\begin{tabular}{|c|c|c|c|c|c|}
\hline Epoch & $\mathrm{OOT}_{i}$ & $T_{14}[\mathrm{mins}]$ & TDV [mins] & $\tau_{i}\left[\mathrm{BJD}_{\mathrm{UTC}}\right]$ & TTV [mins] \\
\hline-2 & $0.999716_{-0.000043}^{+0.000043}$ & $249_{-20}^{+18}$ & $-2_{-20}^{+18}$ & $2454986.0935_{-0.0017}^{+0.0018}$ & $21.2_{-24}^{+2.5}$ \\
\hline-1 & $9043.31_{-0.55}^{+0.57}$ & $239.3_{-8.0}^{+17.5}$ & $-11.3_{-8.0}^{+17.5}$ & $2455019.6976_{-0.0015}^{+0.0016}$ & $25.1_{-2.2}^{+2.4}$ \\
\hline 0 & $1.000152_{-0.000037}^{+0.000038}$ & $242.3_{-5.4}^{+7.0}$ & $-8.3_{-5.4}^{+7.00}$ & $2455053.2943_{-0.0013}^{+0.0013}$ & $18.4_{-1.9}^{+1.9}$ \\
\hline 1 & $0.995332_{-0.000040}^{+0.000039}$ & $255_{-12}^{+19}$ & $5_{-12}^{+19}$ & $2455086.8629_{-0.0015}^{+0.0015}$ & $-28.7_{-2.1}^{+2.2}$ \\
\hline 2 & $0.999948_{-0.000033}^{+0.000035}$ & $256.5_{-7.8}^{+18.4}$ & $6.0_{-7.8}^{+18.4}$ & $2455120.4391_{-0.0014}^{+0.0013}$ & $-64.9_{-2.0}^{+1.9}$ \\
\hline 3 & $1.00403_{-0.00011}^{+0.00011}$ & $246.5_{-8.4}^{+10.8}$ & $-4.0_{-8.4}^{+10.8}$ & $2455154.0749_{-0.0020}^{+0.0019}$ & $-15.3_{-2.9}^{+2.8}$ \\
\hline 4 & $0.999660_{-0.000038}^{+0.000037}$ & $249.3_{-6.3}^{+10.5}$ & $-1.3_{-6.3}^{+10.5}$ & $2455187.6856_{-0.0014}^{+0.0014}$ & $-1.9_{-2.0}^{+2.0}$ \\
\hline 5 & $0.999811_{-0.00035}^{+0.00034}$ & $251.7_{-5.9}^{+9.8}$ & $1.2_{-5.9}^{+9.8}$ & $2455221.3355_{-0.0012}^{+0.0012}$ & $68.0_{-1.8}^{+1.8}$ \\
\hline 6 & $1.000453_{-0.000033}^{+0.000033}$ & $244.3_{-5.5}^{+8.9}$ & $-6.2_{-5.5}^{+8.9}$ & $2455254.9051_{-0.0012}^{+0.0012}$ & $22.4_{-1.7}^{+1.0}$ \\
\hline 7 & $0.999924_{-0.000043}^{+0.000043}$ & $270_{-33}^{+22}$ & $20_{-33}^{+22}$ & $2455288.4673_{-0.0019}^{+0.0000}$ & $-34.0_{-2.7}^{+2.9}$ \\
\hline 8 & $0.999868_{-0.000037}^{+0.000035}$ & $254.2_{-5.4}^{+9.9}$ & $3.7_{-5.4}^{+9.9}$ & $2455322.0599_{-0.0012}^{+0.0012}$ & $-46.7_{-1.8}^{+1.7}$ \\
\hline 9 & $0.999741_{-0.000041}^{+0.000041}$ & $247.6_{-6.8}^{+8.9}$ & $-2.9_{-6.8}^{+8.9}$ & $2455355.6657_{-0.0015}^{+0.0015}$ & $-40.3_{-2.2}^{+2.0}$ \\
\hline 10 & $0.999855_{-0.00039}^{+0.000038}$ & $242.3_{-7.4}^{+12.4}$ & $-8.3_{-7.4}^{+12.4}$ & $2455389.3435_{-0.0014}^{+0.0014}$ & $69.8_{-2.0}^{+2.0}$ \\
\hline 11 & $0.999786_{-0.000035}^{+0.000036}$ & $267_{-20}^{+17}$ & $16_{-20}^{+17}$ & $2455422.9284_{-0.0014}^{+0.0014}$ & $46.2_{-2.0}^{+2.0}$ \\
\hline 12 & $1.000123_{-0.000040}^{+0.000040}$ & $251.2_{-8.0}^{+13.5}$ & $0.7_{-8.0}^{+13.5}$ & $2455456.4856_{-0.0014}^{+0.0015}$ & $-17.4_{-2.0}^{+2.2}$ \\
\hline
\end{tabular}

Table S4: Epoch-specific fitted parameters for the KOI-872 system. Results computed from the weighted posteriors resulting from model $\mathcal{M}_{V}$, using MultiNest. TDVs relative to median duration of all duration realizations in $\mathcal{M}_{V} ; T_{14}=250.5$ mins. Physical system parameters are provided in Table 1.

\begin{tabular}{llll}
\hline \hline Hypothesis & $\log \mathcal{Z}$ & $\sigma$ Preference over $\mathcal{H}_{P}$ & Blend analysis conclusion \\
\hline $\mathcal{H}_{P}$ & $(12000.13 \pm 0.37)$ & - & Plausible \\
$\mathcal{H}_{E B, 33.6}^{c}$ & $(12000.95 \pm 0.37)$ & $(0.8 \pm 0.4)$ & Plausible \\
$\mathcal{H}_{E B, 33.6}^{e}$ & $(12004.29 \pm 0.35)$ & $+(2.4 \pm 0.2)$ & Plausible \\
$\mathcal{H}_{E B, 67.2}^{c}$ & $(11977.45 \pm 0.39)$ & $-(6.41 \pm 0.08)$ & Excluded \\
$\mathcal{H}_{E B, 67.2}^{e}$ & $(11867.05 \pm 0.36)$ & $-(16.13 \pm 0.03)$ & Excluded \\
\hline \hline
\end{tabular}

Table S5: Bayesian evidences for a blend family of hypotheses fitted to the KOI-872 photometry. Out of the various blend scenarios tried, we find the light curve shape has no significant preference between hypotheses $\mathcal{H}_{P}, \mathcal{H}_{E B, 33.6}^{c}$ and $\mathcal{H}_{E B, 33.6}^{e}$. 